\title{
ARTICLE
}

\section{Spaceflight and simulated microgravity suppresses macrophage development via altered RAS/ERK/NFkB and metabolic} pathways

\author{
Lu Shi $\mathbb{B}^{1,2}$, Hongling Tian ${ }^{1,2}$, Peng Wang ${ }^{1,2}$, Ling $\mathrm{Li}^{1,2}$, Zhaoqi Zhang ${ }^{1,2}$, Jiayu Zhang ${ }^{1,2}$ and Yong Zhao (iD)
}

Spaceflight-associated immune system weakening ultimately limits the ability of humans to expand their presence beyond the earth's orbit. A mechanistic study of microgravity-regulated immune cell function is necessary to overcome this challenge. Here, we demonstrate that both spaceflight (real) and simulated microgravity significantly reduce macrophage differentiation, decrease macrophage quantity and functional polarization, and lead to metabolic reprogramming, as demonstrated by changes in gene expression profiles. Moreover, we identified RAS/ERK/NFKB as a major microgravity-regulated pathway. Exogenous ERK and NFKB activators significantly counteracted the effect of microgravity on macrophage differentiation. In addition, microgravity also affects the p53 pathway, which we verified by RT-qPCR and Western blot. Collectively, our data reveal a new mechanism for the effects of microgravity on macrophage development and provide potential molecular targets for the prevention or treatment of macrophage differentiation deficiency in spaceflight.

Keywords: Microgravity; Hematopoietic progenitor cells; Macrophage differentiation; Macrophage polarization; RAS/ERK/NFKB pathway

Cellular \& Molecular Immunology (2021) 18:1489-1502; https://doi.org/10.1038/s41423-019-0346-6

\section{INTRODUCTION}

Since the beginning of space travel, there have been reports of the deleterious effects of spaceflight on human health. Spaceflight involves multiple biological stressors, including microgravity, radiation, loss of the light-dark cycle, and confinement. Among these, microgravity (between $10^{-3}-10^{-5} \mathrm{~g}$ ) exposure has been reported to have the strongest effects on human physiology and psychology. ${ }^{1,2}$ Space research over the past 50 years has demonstrated that during and after spaceflight, a series of changes occur in human blood, including anemia, thrombocytopenia, reduced lymphocyte numbers, suppressed lymphocyte function, and red blood cell structural abnormalities. ${ }^{3,4}$ Moreover, published reports have documented that cosmonauts develop more opportunistic and reoccurring infections than healthy ground control individuals, likely resulting from immune suppression and immune tolerance associated with immune cell dysfunction under microgravity.

Recently, the effects of microgravity on immune function have received increased attention and have been investigated using astronaut leukocytes, ${ }^{5}$ space-flown mice and rats, ${ }^{6,7}$ and in vitro cell culture. ${ }^{8}$ Together, data from the International Space Station, Skylab, and space shuttle suggest that dysregulated immune cell-mediated cytokine secretion is a major mechanism leading to spaceflightassociated immune dysfunction. ${ }^{5,9-12}$ For example, after space travel, shifts occur in circulating cytokines, including increased levels of $\mathrm{T}$ lymphocytes producing IL-4, IL-8, IL-17, and IFN- $\gamma^{8,13,14}$ and decreased production of IL-1, IL-6, IL-12, and TNF-a in monocytes/ macrophages. ${ }^{15-18}$ However, the causes of these immune cell dysfunctions and the associated changes in innate (macrophages and NK cells) and adaptive (T-cell) functions have not been extensively and systematically examined. The monocyte/macrophage system is responsible for the first line of innate immune defense against invading pathogens. It is essential for attacking and killing pathogens as well as for the clearance of apoptotic cells. Thus, maintaining a robust innate immune system while in a low-gravity environment is crucial for human health and performance during long-term space missions. Although studies have reported diverse alterations in monocyte/macrophage functions in space or simulated microgravity, including changes in cell morphology, proliferation, growth, differentiation, signal transduction, and gene expression, ${ }^{18-21}$ key altered gene expression networks related to impaired macrophage differentiation and function in microgravity remain unknown.

In the current study, we elucidated the major molecular mechanisms underlying the effects of microgravity on macrophage differentiation from hematopoietic progenitor cells (HPCs). We found that microgravity significantly inhibits the maturation of macrophages and impedes M1/2 polarization after in vitro differentiation compared to macrophages differentiated under normal gravity. Further, we demonstrate that macrophage differentiation deficiency caused by microgravity involves the RAS/ERK/NFKB pathway, the p53 pathway, and alterations in cellular metabolism.

\footnotetext{
${ }^{1}$ State Key Laboratory of Membrane Biology, Institute of Zoology, Chinese Academy of Sciences, Beijing, China; ${ }^{2}$ University of Chinese Academy of Sciences, Beijing, China and ${ }^{3}$ Institute for Stem Cell and Regeneration, Chinese Academy of Sciences, Beijing, China Correspondence: Yong Zhao (zhaoy@ioz.ac.cn)

These authors contributed equally: Lu Shi, Hongling Tian, Peng Wang, Ling Li
}

Received: 9 September 2019 Revised: 12 November 2019 Accepted: 3 December 2019

Published online: 3 January 2020 
L Shi et al.

A

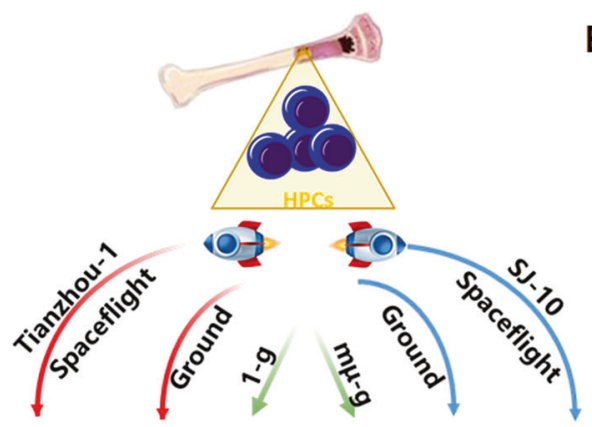

Differentiation

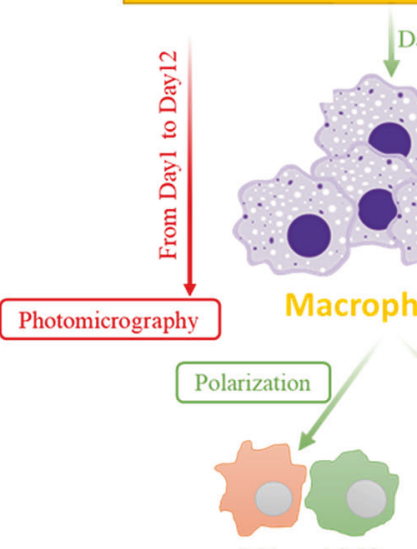

M1 and M2
B

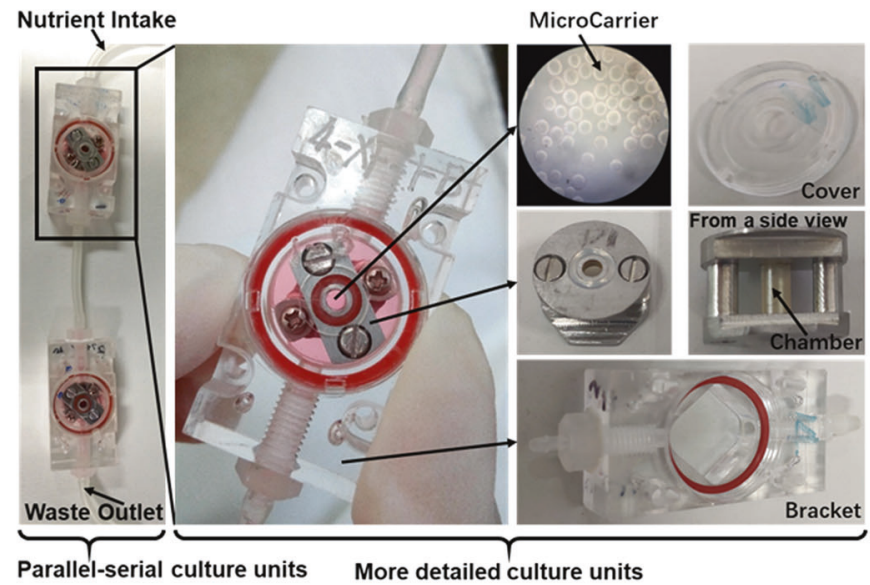

C

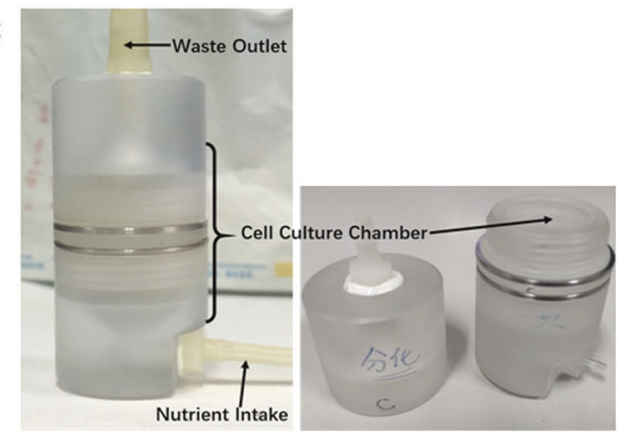

D

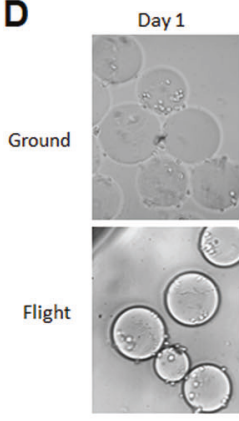

G

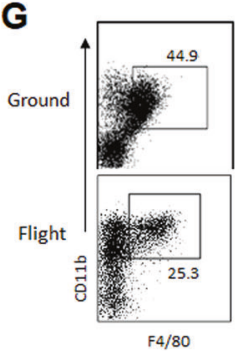

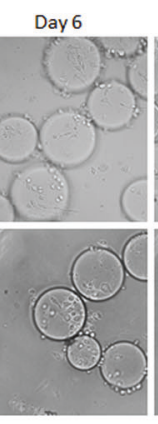

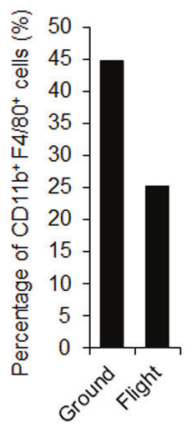

Day 12
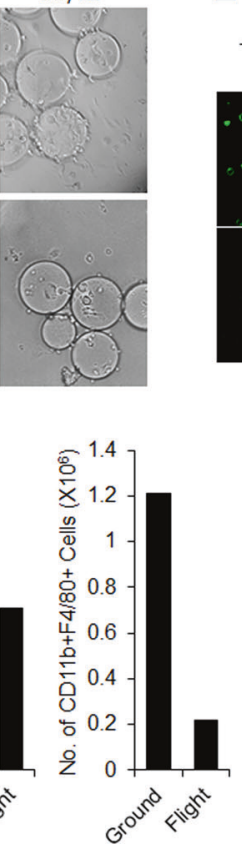

$\mathbf{E}$

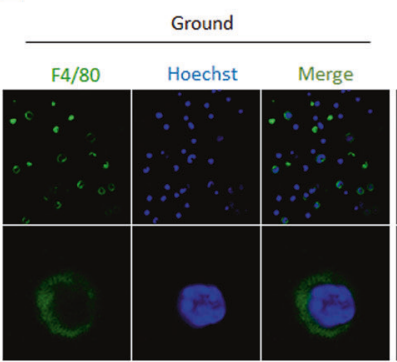

H

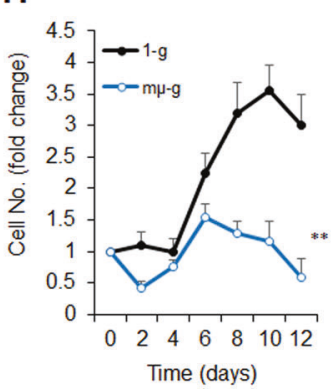

$\mathbf{F}$

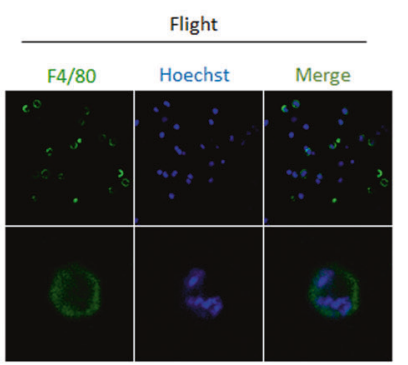

Fig. 1 Microgravity decreases the total number of cells and inhibits the differentiation of macrophages from HPCs. a A schematic of all experimental processes and analyses. $\mathbf{b}$ The cell observation unit used in the Tianzhou- 1 cargo ship is made of polystyrene (PS). c The cell culture unit used in the SJ-10 satellite is made of polycarbonate (PC). d The cell growth state was imaged by the Tianzhou-1 cargo ship program, and the presented result is one representative of three independent samples showing identical results at 20x magnification. e Immunofluorescence staining (4× magnification in the upper figures, $60 \times$ magnification in the lower figures). $\mathbf{f}, \mathbf{g}$ The cell count is of the cells collected from the SJ-10 space program. Spaceflights, including the Tianzhou-1 cargo ship and SJ-10 satellite, are labeled as "Flight", and the ground controls are labeled as "Ground". h Simulated microgravity (labeled "m $\mu-g$ ") inhibits cell proliferation and i macrophage differentiation compared with that achieved under normal gravity (labeled "1-g"). For the simulated microgravity experiments, data are presented as the mean \pm SD of three independent experiments. ${ }^{* *} P<0.01$ compared with the control. 
A

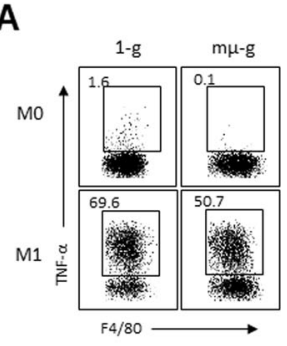

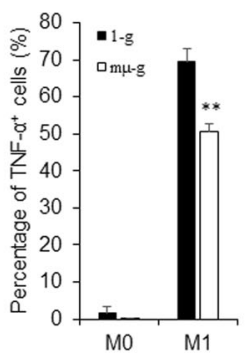

B
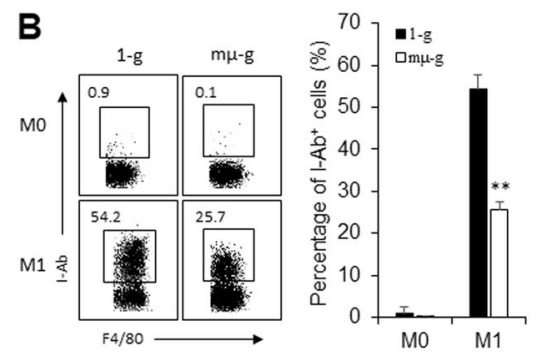

C
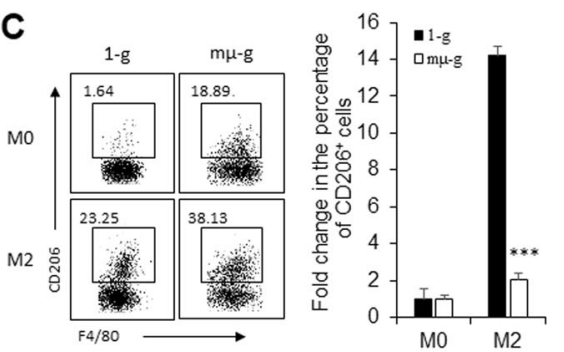
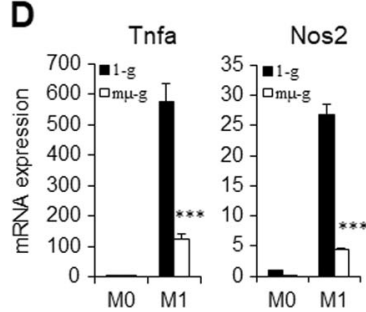

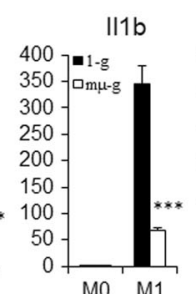

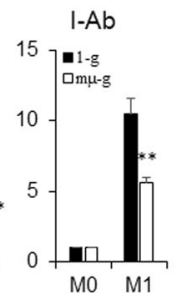
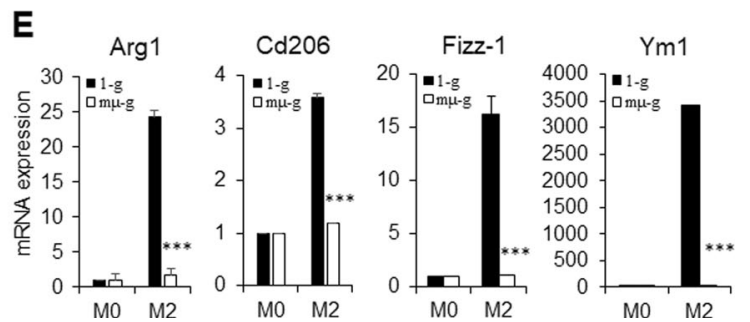

Fig. $2 M 1 / M 2$ macrophage polarization is impaired after in vitro differentiation in a simulated microgravity environment. M1 macrophage polarization is impaired after in vitro differentiation in a simulated microgravity environment (labeled "m $\mu-\mathrm{g}$ "). FACS plot and histograms of macrophages expressing the M1 markers TNF- $\alpha$ (a) and I-Ab (b). M2 macrophage polarization is impaired after in vitro differentiation under simulated microgravity. FACS plot and histograms of macrophages expressing the M2 marker CD206 (c). Relative expression of $\mathrm{M} 1$ polarization genes (d) and $\mathrm{M} 2$ polarization genes (e) in macrophages after in vitro differentiation under simulated microgravity. Data are presented as the mean \pm SD from three independent experiments. ${ }^{* * *} P<0.001$. ${ }^{* *} P<0.01$. Control: macrophages after in vitro differentiation under normal gravity (labeled "1-g").

\section{RESULTS}

Spaceflight and simulated microgravity significantly inhibit the differentiation of macrophages from HPCs

To study the effects of microgravity on macrophage differentiation, mouse HPCs (Lin cells) isolated from bone marrow were stimulated to undergo differentiation using macrophage colony stimulating factor (M-CSF) plus IL-3 and IL-6 under spaceflight (real) and simulated microgravity conditions (Fig. 1a). Two specially designed cell culture devices (Fig. 1b, c), which allow automatic replacement of the culture medium aboard the Tianzhou-1 cargo ship or the SJ-10 recoverable satellite, were used to culture cells. Over 12 days in culture, the HPCs gradually differentiated into macrophages, which was observable during imaging based on their morphological change from being round to flat and polygonal in shape (Fig. 1d). Immunofluorescence staining with an anti-F4/80 antibody further confirmed the presence of macrophages (Fig. 1e). However, after flying on board the SJ-10 satellite in outer space for approximately 12 days, the total cell number was greatly reduced compared with that in ground control samples (Fig. 1f). The images collected from the Tianzhou-1 cargo spacecraft showed that the cells multiplied more slowly during spaceflight than control cells (Fig. 1d). Additionally, a decreased percentage and number of $\mathrm{CD} 11 \mathrm{~b}^{+} \mathrm{F} 4 / 80^{+}$macrophages were observed in the spaceflight cultures (Fig. 1g), suggesting that microgravity inhibits the differentiation of macrophages from HPCs. To confirm this phenomenon, we differentiated macrophages under normal gravity and modeled microgravity conditions for 12 days using a rotary cell culture system (RCCS) (Supplementary Fig. 1). The results showed that the macrophage percentage and number were also significantly lower in the modeled microgravity cultures than in the normal gravity cultures $(P<0.01$, Fig. $1 \mathrm{~h}, \mathrm{i})$, indicating that microgravity inhibits the differentiation of HPCs into macrophages.

Simulated microgravity impairs the functional polarization of macrophages

Bone marrow-derived macrophages can be polarized into two functionally distinct phenotypes depending on the cytokine microenvironment: ${ }^{22}$ proinflammatory/classically activated macrophages (M1) and proresolving/alternatively activated macrophages (M2). ${ }^{23,24}$ Both types play important roles in the immunology, tissue homeostasis, and disease pathogenesis of resolving and nonresolving inflammation. Thus, we examined whether microgravity affects the functional polarization of macrophages. After the differentiation of macrophages from HPCs under modeled microgravity or normal gravity conditions, we applied either IFN- $\gamma$ and LPS or IL-4 to stimulate M1 and M2 polarization, respectively, as reported previously. ${ }^{25,26}$ The results showed that both $\mathrm{M} 1$ and $\mathrm{M} 2$ polarization were significantly impaired in macrophages that were differentiated under modeled microgravity (Fig. 2). The expression levels of $M 1$ phenotypic markers, ${ }^{27}$ such as TNF- $a$, I-Ab, iNOS (Nos2), IL-1 $\beta$, and IL-6 (Fig. 2a-c), and M2 phenotypic markers, ${ }^{28}$ such as Arg-1, CD206 (Mrc1), Fizz, and Ym1 (Fig. 2d, e), were all reduced in macrophages developed under microgravity compared with macrophages developed under normal gravity. Thus, macrophages developed under microgravity are less responsive to IFN- $\gamma$ and LPS or IL-4 than macrophages developed under normal gravity. Taken together, these data show that exposure to microgravity during macrophage differentiation can inhibit their future polarization.

Key genes related to macrophage proliferation and differentiation are transcriptionally downregulated under spaceflight and simulated microgravity conditions

To evaluate the effects of microgravity on differentiation-related gene expression patterns, we collected macrophages that had been differentiated over 12 days of spaceflight and ground controls for transcriptome analysis by RNA sequencing (RNA-Seq). In addition, we induced macrophages from HPCs under simulated microgravity and normal gravity and assayed their transcriptomes. We identified a total of 2270 significantly differentially expressed genes (DEGs, corresponding to identified transcripts) in the spaceflight versus ground control groups, of which 1575 genes were upregulated and 695 genes were downregulated (Fig. 3a). A similar comparison of DEGs in the simulated microgravity versus normal gravity groups identified 1267 genes. Of these DEGs, 525 genes were upregulated and 742 genes were downregulated under simulated microgravity (Fig. 3b).

We next performed gene ontology (GO) enrichment analysis for biological processes (BPs) in the identified DEGs. We focused 

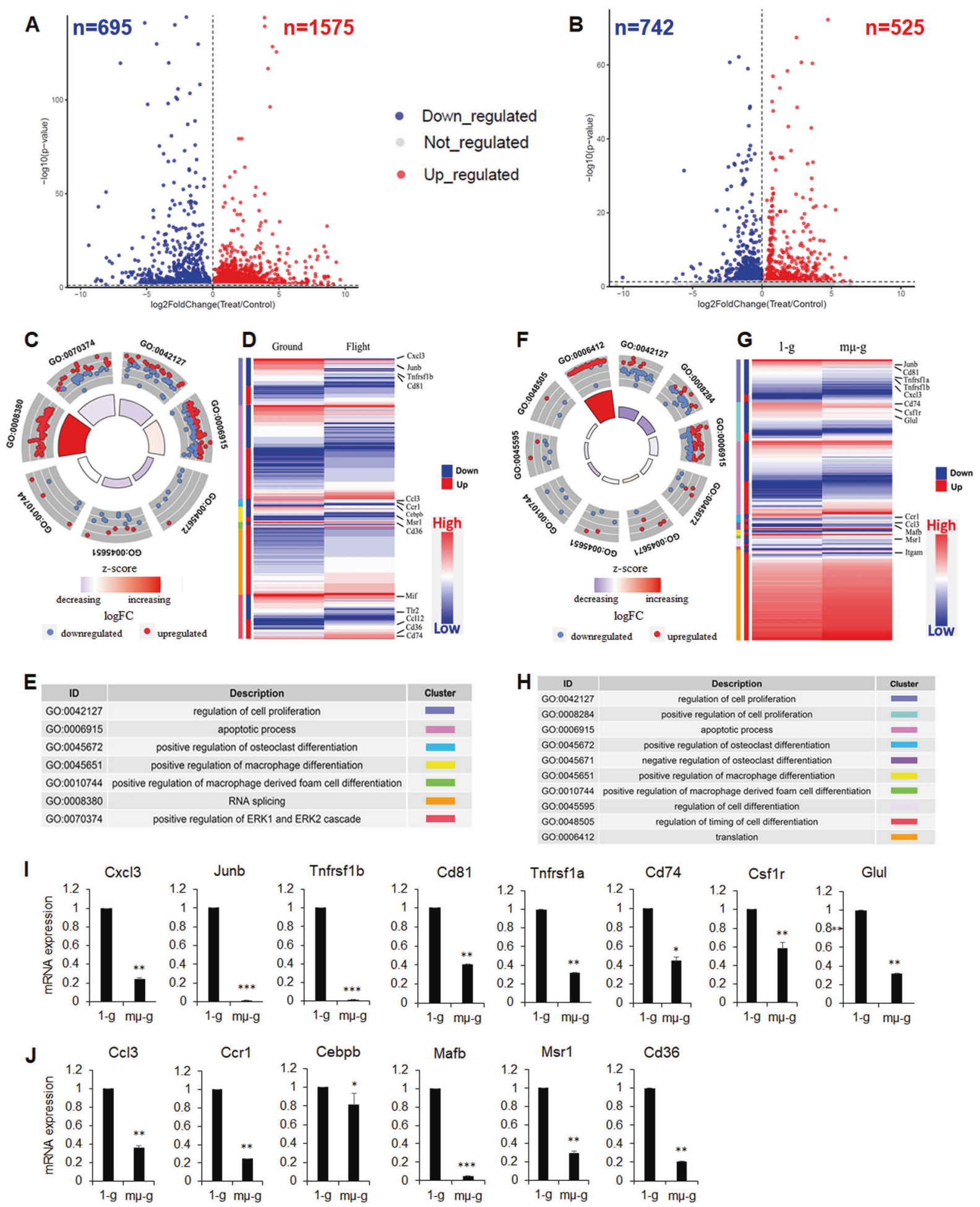

Fig. 3 Microgravity-altered genes involved in key biological processes. Volcano plot of DEGs as a function of statistical significance between microgravity-exposed and control macrophages from spaceflight (a) and ground simulation (b) experiments. c-e From 12 day spaceflight experiments. f-h From simulated microgravity experiments. Circular plots combining the gene expression levels and GO enrichment analyses of genes differentially expressed under microgravity (c and $\mathbf{f}$ ). The logFCs of the assigned genes in each term are shown in scatter plots in the outer circle. The inner circle contains bar plots of the -log10 adjusted P-values, indicating the significance of each term. The color of each bar indicates the GO term z-score. Heatmaps of the gene expression profiles of each GO term (d and $\mathbf{g})$. The labeled genes are validated in $\mathbf{i}$ and $\mathbf{j}$. The tables (e and $\mathbf{h}$ ) represent the detailed information for each $\mathrm{GO}$ term (c and $\mathbf{f}$ ) and cluster (d and $\mathbf{g})$. i Validation of notable genes in the 'cell proliferation' GO cluster by real-time PCR. $\mathbf{j}$ Validation of notable genes in the 'macrophage differentiation' GO cluster by real-time PCR. Data are shown as the means \pm SEM from three PCR assays. Gene expression levels were normalized to those of the housekeeping gene Hprt. Unpaired Student's t-test was used to test for statistical significance ${ }^{*} P<0.05$, ${ }^{* *} P<0.01$, and $\left.{ }^{* * *} P<0.001\right)$. Data from 12 day spaceflight and ground control samples are labeled as "Flight" and "Ground", respectively, and data from simulated microgravity and normal gravity are labeled as " $m \mu-g$ " and " $1-g$ ", respectively. 
on GO terms related to the quantity and differentiation of macrophages. The key BPs, such as cell proliferation, apoptosis, and differentiation, are represented in Fig. 3 and in Supplementary Tables 1 and 2. Four pathways related to proliferation and differentiation were significantly downregulated, while "apoptotic processes" and "RNA splicing" were overrepresented in macrophages differentiated in spaceflight (Fig. 3c-e). Similarly, in macrophages differentiated in simulated microgravity, five major pathways related to proliferation and differentiation were significantly downregulated, but "translation" and "negative regulation of osteoclast differentiation" were upregulated (Fig. 3f-h). These key BP gene sets were subjected to an annotated hierarchical clustering procedure and presented as heatmaps (Fig. 3d, g). Additionally, we validated the relative abundances of some of the most interesting genes using RTqPCR (gene symbols labeled in Fig. $3 \mathrm{~d}, \mathrm{~g}$ ). The results confirmed that $C x C l 3$, Junb, Tnfrsf1b, Tnfrsf1a, and $C d 81(P<0.01$, Fig. 3i, associated with "regulation of cell proliferation") and Cd74, Csf1r, and Glul (Fig. 3i, associated with "positive regulation of cell proliferation") were downregulated. Ccl3, Ccr1, Mafb, and Cebpb (associated with "positive regulation of osteoclast differentiation") and Msr1 and Cd36 (associated with "positive regulation of macrophage differentiation") were also significantly decreased $(P<0.01$, Fig. $3 \mathrm{j})$. These results provide mechanistic insight into how microgravity decreases the differentiation of HPCs into macrophages.

Microgravity-induced alterations in cellular metabolism

We tried to use the DEGs from our transcriptome analysis to identify microgravity-induced metabolic changes during macrophage differentiation. However, we found that the DEGs in these two experiments were very limited due to the small sample size (Fig. 4a, b). Thus, for further analysis, we included spaceflightassociated DEGs that followed a similar up-/downward trend in the microgravity-simulated experiment even if they were not statistically significant. Including these genes, the shared downregulated genes accounted for $69.6 \%$ of all the downregulated genes (Fig. 4c), while the shared upregulated genes accounted for 48.4\% (Fig. 4d). These genes were used as input for further metabolic pathway analysis.

The first level of the Kyoto Encyclopedia of Genes and Genomes (KEGG) has 7 main categories, ${ }^{29}$ including metabolism (the first category), which summarizes all annotated metabolic pathways in biological systems. According to the "KEGG Pathway Maps", metabolic pathways include 11 subcategories, which can be visualized in a global map (map numbers 01100 s) with different colors. To analyze metabolic changes under spaceflight microgravity, we separately annotated each input gene set by mapping it to known metabolic pathways in the KEGG database and identifying significantly enriched pathways. After comparative analysis, we identified 8 subclasses of KEGG-enriched metabolic pathways (Fig. 4e), including carbohydrate metabolism, lipid metabolism, nucleotide metabolism, and amino acid metabolism, labeled 1.1-1.9 (subclass 1.2 was not enriched in our data). Our results showed that carbohydrate metabolism was downregulated by microgravity (except "butanoate metabolism"), but lipid and nucleotide metabolism were upregulated (Fig. 4e). There was no consistent trend in amino acid metabolism, as some key genes went up, and others went down (Fig. 4e). However, both "vitamin B6 metabolism" and "terpenoid backbone biosynthesis" were clearly enriched in the upregulated pathway (Fig. 4e). To gain further insight into microgravity-induced metabolic changes, the $\log _{2}$ fold change $\left(\log _{2} F C\right)$ values of fragments per kilobase per million mapped reads (FPKM) for key enzymes involved in specific metabolic pathways were extracted and displayed in a heatmap (Fig. 4f). This analysis showed that microgravity was associated with decreased expression levels of ten key glycolysis enzymes, including hexokinase (Hk1 and $\mathrm{Hk} 2)$ and fructose-6-phosphate kinase (Pfk1 and Pfkp) (Fig. 4f). These are the most important carbohydrate metabolism regulatory enzymes, and they are rate-limiting in multiple carbon metabolism pathways. In addition, upregulated genes such as fatty acid synthase (Fasn), hydroxymethylglutaryl-CoA synthase (Hmgcs1), stearoyl-CoA desaturase (scd1/2), and acyl-CoA synthetases (Acs/3) are involved in lipid metabolism pathways. All microgravityinduced changes in central metabolic pathways are displayed in Supplementary Fig. 2 and Supplementary Fig. 3, which are based on global maps (map numbers $01100 \mathrm{~s}$ ).

The metabolic changes associated with real microgravity (spaceflight) were very similar to those resulting from simulated microgravity. To verify this, the expression levels of Hk1, Pfkp, Fasn, and $A c s / 3$ and their corresponding protein products were detected by RT-PCR and Western blot, respectively, after simulated microgravity exposure. We also determined the expression of $H$ k2, Hmgcs 1, and Scd 1 by RT-PCR. These results showed that key metabolic genes and proteins were downregulated under simulated microgravity (Fig. 4g, h, k, I). In addition, glycolysis levels and fatty acid oxidation (FAO) dependency were measured using Agilent seahorse XF. Compared with those differentiated under normal gravity, macrophages differentiated under simulated microgravity for 12 days had substantially lower basal glycolysis levels (Fig. 4i, j), and their mitochondria were more dependent on FAO (Fig. $4 \mathrm{~m}$ ). Together, these results indicate that exposure to microgravity during differentiation causes cellular metabolism reprogramming.

\section{Major signaling pathways inhibited by microgravity during} macrophage differentiation

To determine the molecular mechanisms underlying microgravityrelated changes in macrophage development, we performed gene set enrichment analysis (GSEA) of BioCarta and KEGG pathways. This analysis highlighted potential roles of the RAS, ERK (originally called MAPK), and NFKB signaling pathways, which all involve some of the genes downregulated by microgravity. GSEA can determine whether an a priori defined set of genes shows statistically significant and concordant differences between two biological states. We combined gene sets from the RAS, ERK, and NFKB signaling pathways to generate a new gene set, the RAS/ ERK/NFKB pathway, and performed GSEA using this gene set to compare the normal gravity (ground control) and microgravity (spaceflight and simulated microgravity) groups. The GSEA plot showed that the microgravity samples tended to express lower levels of genes in the RAS/ERK/NFKB signaling pathway than the normal gravity samples (Fig. 5a). The detailed GSEA analysis of each sample is presented as a heatmap (Fig. 5b), which shows that most of the key genes were simultaneously downregulated; however, a few of the key genes were uniquely downregulated in spaceflight or simulated microgravity conditions. Therefore, we validated several genes from this pathway by qRT-PCR (Fig. $5 \mathrm{c}$ ) and confirmed that all of these genes were significantly downregulated under simulated microgravity. The integrated RAS/ERK/ NFKB pathway model, represented graphically in Fig. $5 d$, is clearly a major signaling pathway regulating macrophage differentiation, proliferation, and function. This result suggests that genes in the RAS/ERK/NFKB pathway may have important roles in mediating microgravity-related changes in the survival, proliferation, differentiation, and even functional polarization of macrophages, perhaps by a cascade downstream of colony stimulating factor 1 receptor (CSF-1R).

Activation of ERK and NFKB rescues the inhibitory effects of microgravity on macrophage development by metabolic conversion

To determine the importance of the RAS/ERK/NFKB pathway in microgravity-impaired macrophage differentiation, we added an 
A

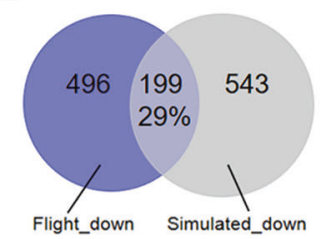

B

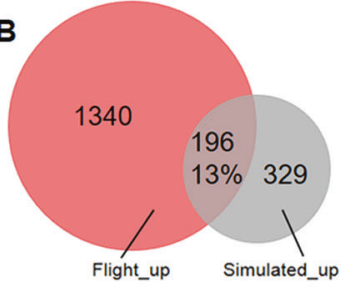

E

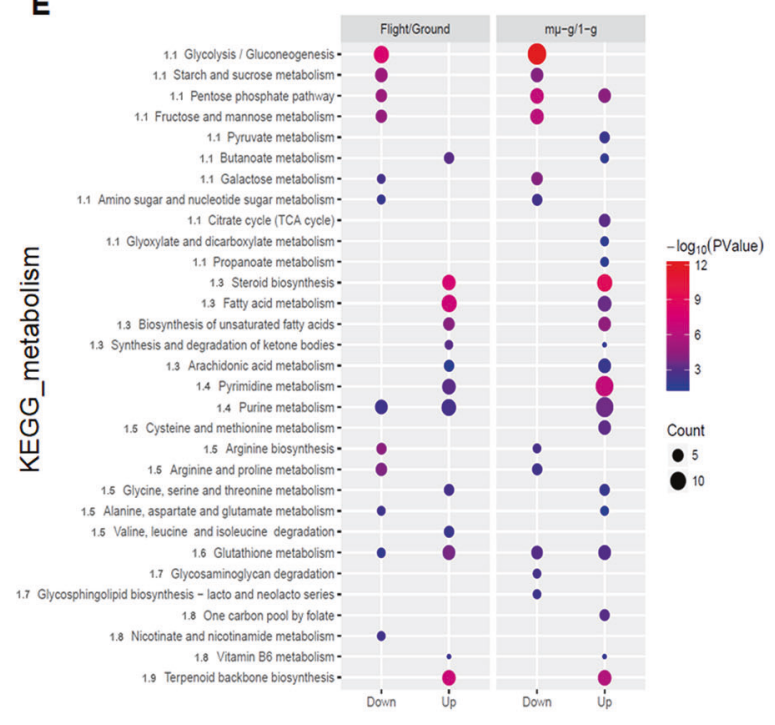

C

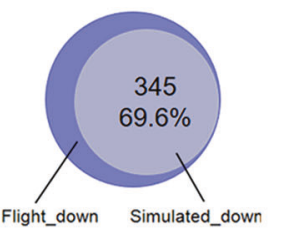

Simulated_down

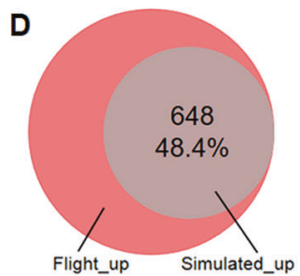

F Flight $m \mu-g$

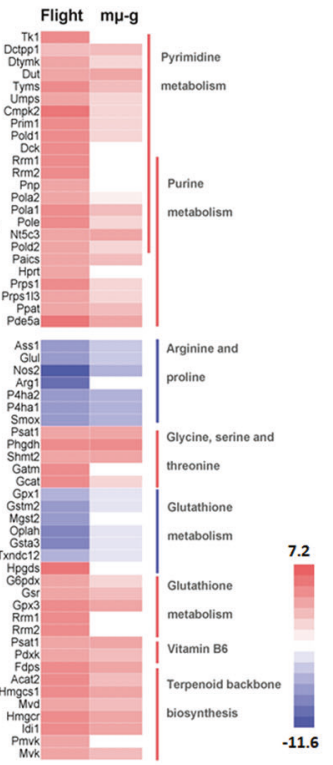

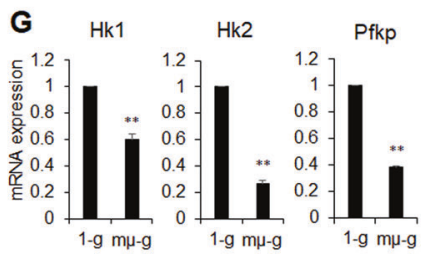

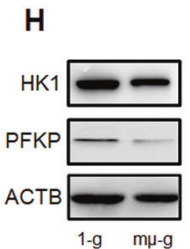

K
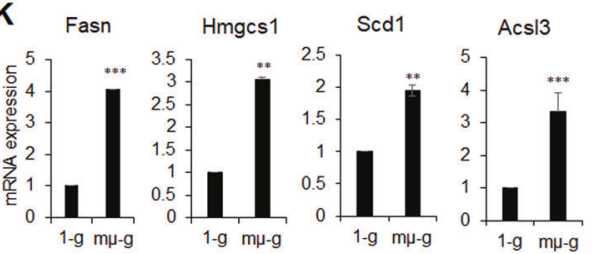

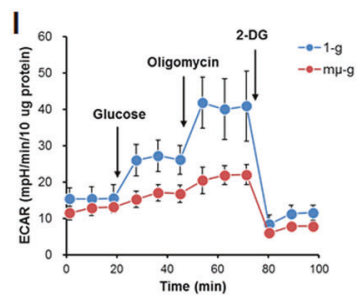

L

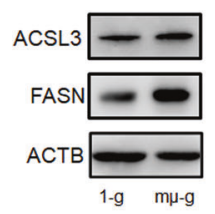

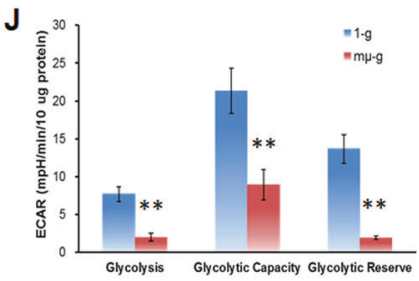

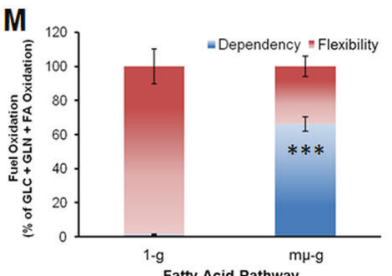

Fig. 4 Metabolic changes in response to microgravity. Venn diagram illustrating the overlap of significantly upregulated (a) and downregulated (b) genes between the spaceflight and ground simulation samples. Venn diagram illustrating the overlap of statistically significant upregulated (c) and downregulated (d) genes in the spaceflight experiment; the genes followed similar up-/downward trends in the simulated experiment without consideration of statistical significance. For all Venn diagrams (a-d), the cardinality of sets and intersection sets is represented by their corresponding circle sizes, and the percentage represents the percentage of intersection genes accounting for genes selected in the spaceflight experiment. e-f Summary of metabolic pathways enriched for DEGs in the 'flight' (spaceflight samples) versus 'ground' (ground control samples) groups and ' $m \mu-g$ ' (simulated microgravity samples) versus ' $1-g^{\prime}$ ' (normal gravity samples) groups. e A dotplot representing the degrees of enrichment for all metabolic pathways. Up- and downregulation of pathways in the spaceflight and simulation experiments are plotted on the $x$-axis. The dot size represents the gene count, and the dot color represents the $P$-value. KEGG subclasses 1.1-1.9 are labeled on the left of each metabolic pathway name. $\mathbf{f}$ Heatmaps displaying the log ${ }_{2} \mathrm{FCs}$ of specific genes in each metabolic pathway. Empty values are white. g Real-time PCR for notable genes in the glycolysis pathway. Data are shown as the mean \pm SEM of three PCR assays. Gene expression levels were normalized using $\beta$-Actin. An unpaired Student's $t$-test was used to test for statistical significance ${ }^{* *} P<0.01$ and $\left.{ }^{* * *} P<0.001\right)$. $\mathbf{h}$ Validation of key enzymes in the glycolysis pathway by Western blot. i Data from the functional glycolysis assay obtained using the Seahorse XF24 Analyzer. At the indicated times, glucose, oligomycin, and 2-deoxyglucose (2-DG) were injected at final concentrations of $10 \mathrm{mM}, 1 \mu \mathrm{M}$, and $50 \mathrm{mM}$, respectively. $\mathbf{j}$ Basal glycolysis, glycolysis capacity, and glycolysis reserve values were calculated from the glycolysis assay in $\mathbf{I}, n=3,{ }^{* *} P<0.001$. $\mathbf{k}-\mathbf{m}$ Validation of the microgravity-induced fatty acid metabolic pathway. k Real-time PCR for notable genes in the fatty acid metabolic pathway. Data are shown as the mean \pm SEM of three PCR assays. Gene expression levels were normalized to $\beta$-Actin. An unpaired Student's t-test was used to test for statistical significance $(* * P<0.01$ and $\left.{ }^{* * *} P<0.001\right)$. I Validation of key enzymes in the fatty acid metabolic pathway by Western blot. $\mathbf{m}$ Fatty acid metabolism as a percentage of total mitochondrial respiration measured with the Seahorse XF24 Analyzer using the Mito Fuel Flex Test Kit, $n=3$ (***P $\leq 0.001)$. 
A

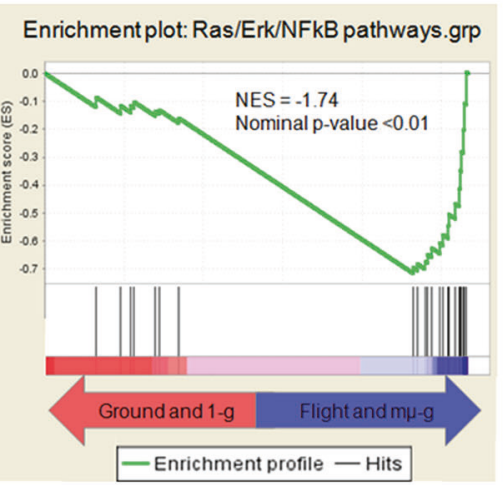

B
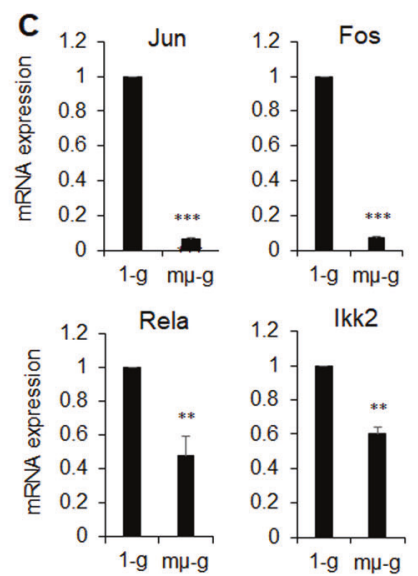
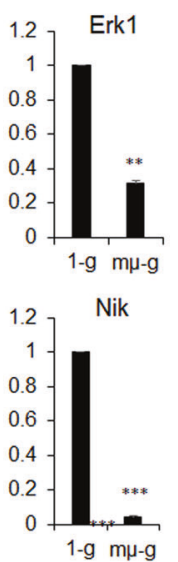

D
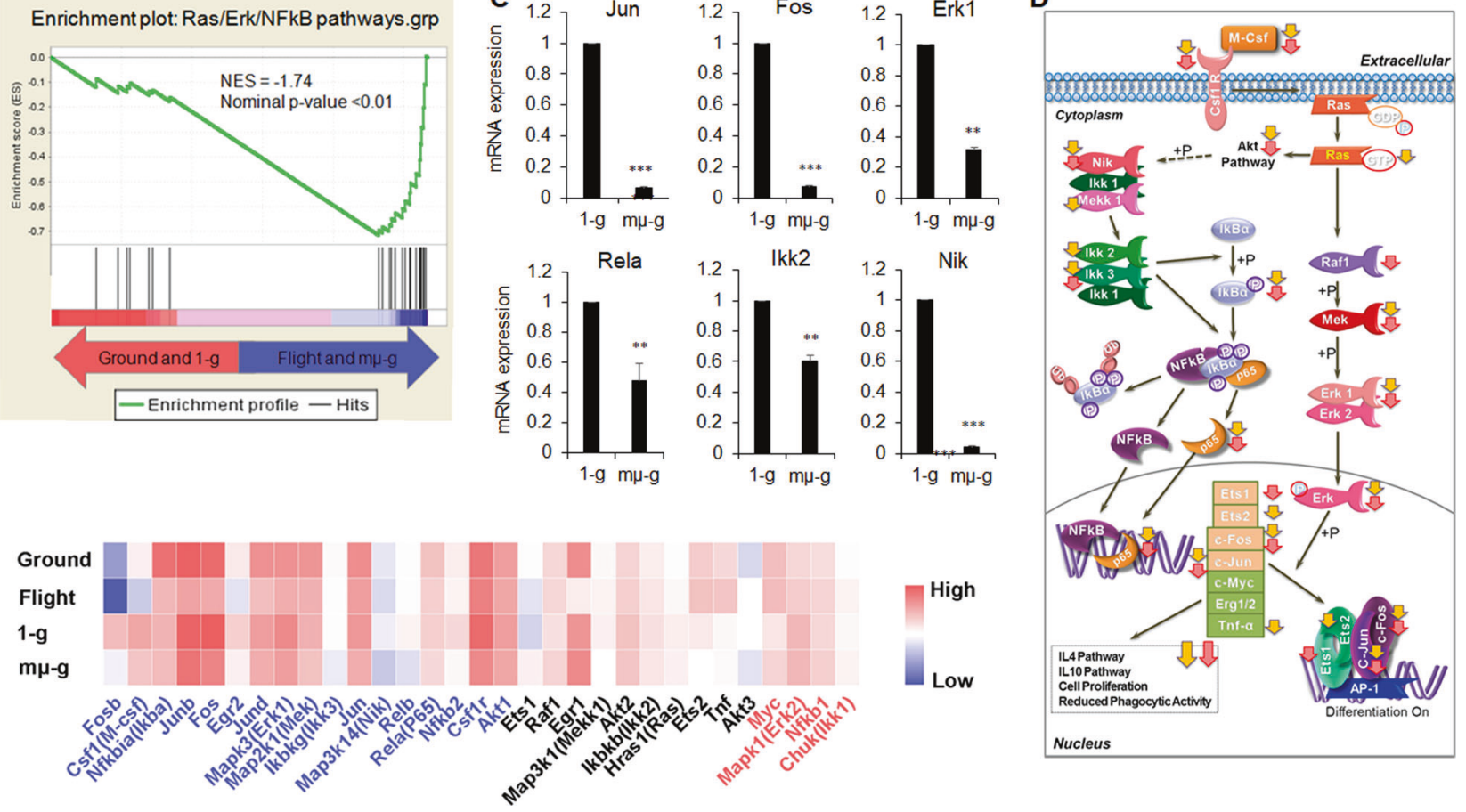

Fig. 5 The major macrophage differentiation signaling pathways impacted by microgravity. KEGG and Biocarta pathway annotations were obtained from mSigDB. A number of genes associated with the RAS/ERK/NFKB pathways were downregulated in the microgravity samples. a GSEA plot showing the RAS/ERK/NFKB pathway analysis comparing control and microgravity-treated samples. The plot shows profiles of the running enrichment score and positions of gene set members on the ranked list of genes. NES, normalized enrichment score. $\mathbf{b}$ Heatmap of gene expression values from the RAS/ERK/NFkB pathways. Blue gene symbols represent shared downregulated genes. Black gene symbols represent genes uniquely downregulated in the spaceflight or simulated microgravity experiments. Red gene symbols represent shared upregulated genes. c Validation of notable genes in the RAS/ERK/NFKB signaling pathways. mRNA expression was determined by qRT-PCR. Data from three PCR assays are shown as the mean \pm SEM. Gene expression levels were normalized using Hprt. An unpaired Student's t-test was used to test for statistical significance $\left({ }^{* *} P<0.01,{ }^{* * *} P<0.001\right)$. d Microgravity-inhibited integrative RAS/ERK/NFKB pathway model. Enzymes, substrates, and products are represented as modules, and the activated reactions are represented as arrows. The expression profiles of all genes in this pathway are represented by up and down arrows. Orange arrows represent changes in the spaceflight samples, and pink arrows indicate changes in simulated microgravity samples. Data from 12 day spaceflight and ground control samples are labeled as "Flight" and "Ground", respectively, and data from simulated microgravity and normal gravity samples are labeled as "m $\mu-\mathrm{g}$ " and "1-g", respectively.

ERK agonist (LM22B-10) or an NFKB agonist (doxifluridine) to the culture medium under simulated microgravity and analyzed macrophage differentiation. We found that the ERK agonist returned the macrophage differentiation ratio back to approximately $60 \%$ (Fig. 6a). To further demonstrate that the ERK signaling pathway is altered during macrophage differentiation under microgravity, we assessed the phosphorylation levels of transcription factors downstream of ERK (c-FOS and c-JUN). The expression levels of these transcription factors were decreased by simulated microgravity but restored by the ERK agonist (Fig. 6b). Additionally, the levels of both total and phosphorylated ERK were also markedly lower under simulated microgravity than under normal gravity but not after application of the ERK agonist (Fig. 6b). This result indicates that the phosphorylation and activation of ERK can increase the phosphorylation of c-FOS and c-JUN, counteracting the effect of microgravity on macrophage differentiation. Furthermore, the results of glycolysis and FAO analysis (Fig. 6c, d) and real-time PCR analysis (Fig. 6e, f) showed that the ERK agonist partially rescued the effect of microgravity on the glycolysis pathway (Fig. 6c, e) but failed to counteract the effect of microgravity on FAO (Fig. 6d, f).

Similarly, the NFKB agonist also rescued macrophage differentiation under microgravity (Fig. 6g). The expression levels of target transcription factors (c-FOS and c-JUN), nuclear factor NFKB p65 subunit, and an upstream molecule, IKBA, were either not affected or slightly increased by the NFKB agonist in normal gravity but were markedly increased by the NFKB agonist under simulated microgravity (Fig. 6h). These results suggest that the macrophage induction medium (which includes M-CSF plus IL-3 and IL-6) may be optimal for the activation of NFKB and transcription of c-FOS and c-JUN in normal gravity but not under microgravity conditions. Additionally, activation of NFKB signaling partially counteracted the effect of microgravity on the glycolysis pathway (Supplementary Fig. 4A, C) but did not counteract the effect of microgravity on FAO (Supplementary Fig. 4B, D). Thus, the ERK and NFKB signaling pathways are involved in the impaired development and glycolysis of macrophages under microgravity.

Functional assessment of macrophage differentiation regulatory networks impacted by microgravity

To investigate other pathways in macrophages potentially altered by microgravity, we performed GSEA of KEGG pathway analysis using DEGs in the microgravity and normal gravity groups. The top 20 most significantly different pathways are displayed in Supplementary Fig. 6A (the DEG FPKMs are shown in Supplementary Table 3). We found that the upregulated genes were mainly enriched in the gene expression, gene regulation, repair and degradation of genes and proteins, and protein export pathways (Supplementary Fig. 5A), which are all pathways that have some signaling crosstalk (Supplementary Fig. 5B). However, the downregulated genes were not only enriched in the abovementioned NFKB and MAPK pathways but also in the intercellular and extracellular matrix interactions, endocytosis, and circadian rhythm pathways (Supplementary Fig. 5A), which also have 
A

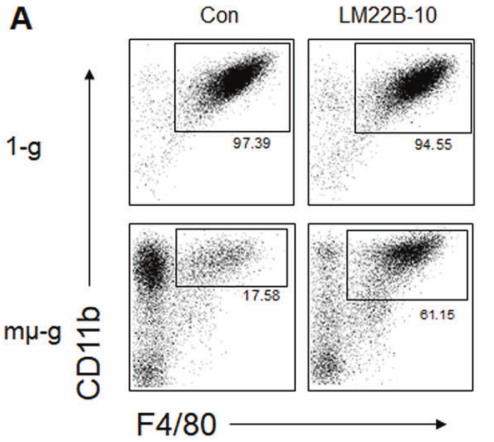

C
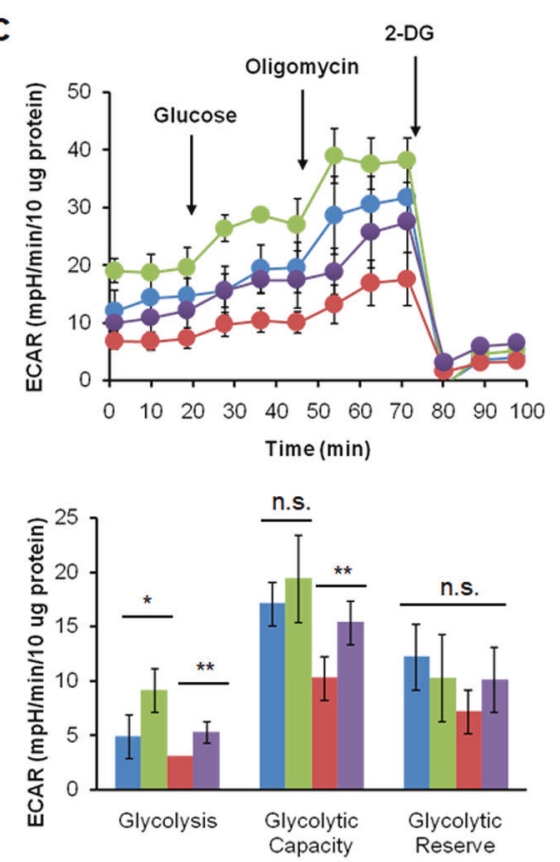

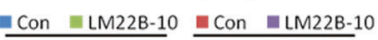

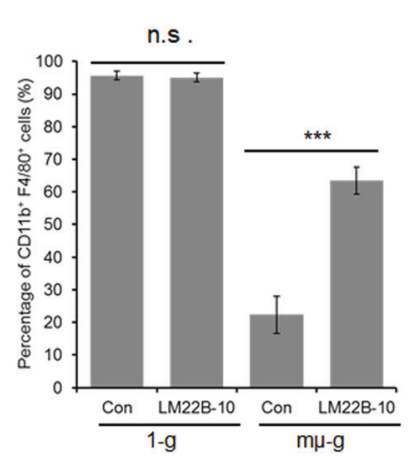

B

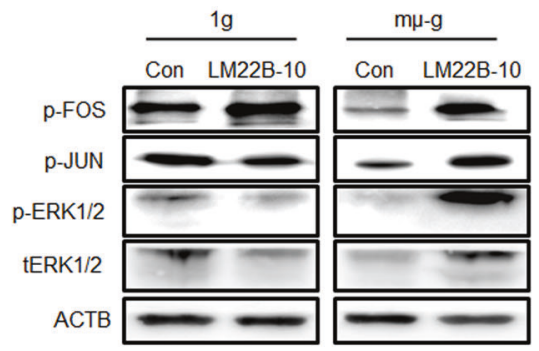

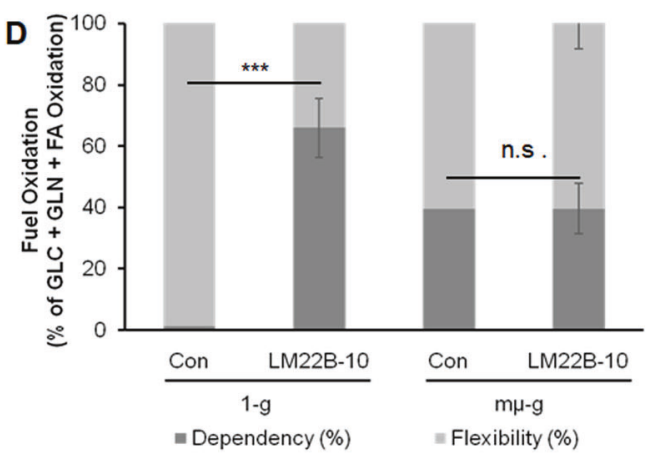
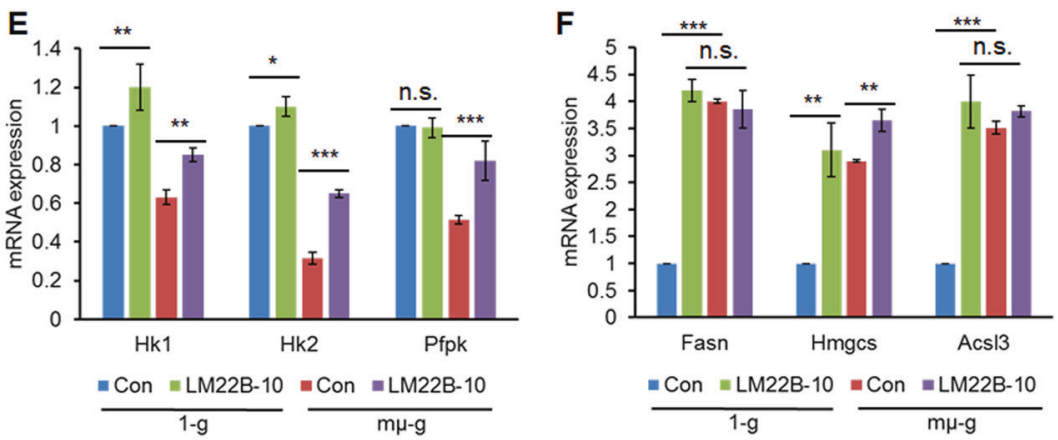

G

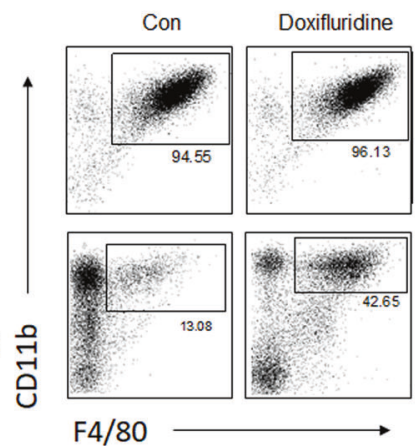

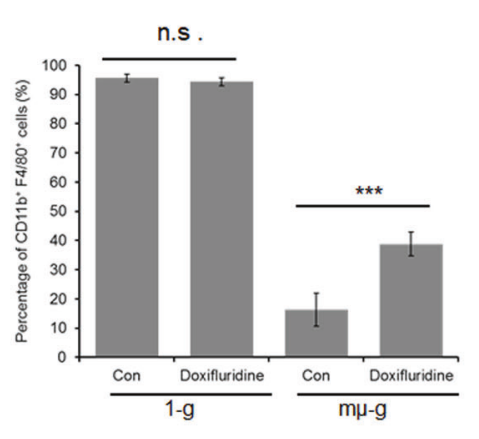

H

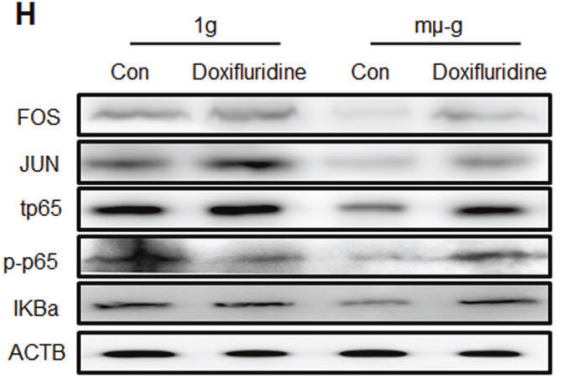

Fig. 6 Activation of RAS/ERK/NFKB signaling pathways rescues the effects of microgravity. Activation of the ERK signaling pathway partially rescues the effects of microgravity. HPCs cultured in normal gravity (labeled as " $1-\mathrm{g}^{\text {") }}$ or simulated microgravity (labeled as "m $\mu-\mathrm{g}^{\text {") }}$ for 12 days without (labeled as "Con") or with LM22B-10 (activator of ERK, labeled as "LM22B-10") were analyzed. a Representative FACS analysis of macrophage differentiation. b Immunoblot analysis of the phosphorylation of c-FOS (p-FOS), c-JUN (p-JUN), and ERK1/2 (p-ERK1/2) as well as total ERK1/2 (tERK1/2). c Functional glycolysis measured with the Seahorse XF24 Analyzer. At the indicated times, glucose, oligomycin, and 2-DG were injected at final concentrations of $10 \mathrm{mM}, 1 \mu \mathrm{M}$, and $50 \mathrm{mM}$, respectively. Basal glycolysis, glycolysis capacity, and glycolysis reserve values were calculated from the glycolysis assay, $n=3, * P<0.05$ and ${ }^{* *} P<0.001$. d Fatty acid metabolism as a percentage of total mitochondrial respiration measured with the Seahorse XF24 Analyzer using the Mito Fuel Flex Test Kit, $n=3\left({ }^{* * *} P \leq 0.001\right)$. e Real-time PCR for notable genes in the glycolysis pathway. Data are shown as the mean \pm SEM from three PCR assays. Gene expression levels were normalized to $\beta$-Actin. An unpaired Student's $t$-test was used to test for statistical significance $\left({ }^{* *} P<0.01\right.$ and $\left.{ }^{* * *} P<0.001\right)$. $f$ Real-time PCR for notable genes in the fatty acid metabolic pathway. Data from three PCR assays are shown as the mean \pm SEM. Gene expression levels were normalized to $\beta$-Actin. An unpaired Student's ttest was used to test for statistical significance ${ }^{* *} P<0.01$ and $\left.{ }^{* * *} P<0.001\right) . \mathbf{g}, \mathbf{h}$ Activation of the NFkB signaling pathway also partially rescues the effects of microgravity. HPCs cultured in normal gravity (labeled as "1-g") or simulated microgravity (labeled as "m $\mu-\mathrm{g}^{\text {") }}$ for 12 days without (labeled as "Con") or without doxifluridine (NFKB activator, labeled as "Doxifluridine") were analyzed. $\mathbf{g}$ Representative FACS analysis of macrophage differentiation. $\mathbf{h}$ Immunoblot analysis of the phosphorylation of p65 (p-p65) as well as the total amounts of c-FOS, c-JUN, p65 (tp65), and IKBA. 
crosstalk (Supplementary Fig. 5C). We verified the expression levels of some genes by RT-PCR (Supplementary Fig. 6A, B).

We noticed that the p53 signaling pathway was also in the top ten most highly expressed pathways. p53 has been described as "the guardian of the genome" because of its role in conserving genetic stability by preventing mutations. ${ }^{30}$ Furthermore, the p53 signaling pathway is directly involved in the regulation and control of protein degradation by the ubiquitin-proteasome system as well as in primary DNA damage and repair mechanisms, cell cycle regulation (specific changes are shown in Supplementary Fig. 7), and apoptosis (specific changes are shown in Supplementary Fig. 8). As such, we would predict that in response to a microgravity environment, the p53 network would be one of the most important upregulated networks in macrophages. Therefore, we analyzed the p53 signaling pathway in detail (Fig. 7). The transcriptional levels of all p53-related genes are presented as a heatmap in Fig. 7a, and a schematic diagram referring to KEGG pathways is displayed in Fig. 7b, highlighting all genes upregulated by microgravity. In addition, the upregulation of some key genes in this pathway was confirmed by RT-PCR (Fig. 7c), and the upregulation of the most important transcription factors regulating cell cycle arrest and apoptosis, CHK1 and BAX, was confirmed by Western blot (Fig. 7d). Thus, the p53 network is likely one of the most important upregulated signaling pathways by macrophages in response to microgravity environments.

\section{DISCUSSION}

In the present study, we demonstrated that microgravity decreased cell numbers, disrupted in vitro macrophage differentiation, and severely impaired macrophage functional polarization (M1/M2). We used next-generation sequencing (NGS) to study microgravity-associated transcriptional changes and identified over- and underrepresented gene signatures in macrophages differentiated under microgravity. The resulting gene expression profiles were consistent with the abnormal macrophage physiology observed in space and under modeled microgravity. Differentially expressed genes were largely related to the RAS/ERK/NFKB pathway and other signaling pathways, such as the p53 pathway. Additionally, gene expression analysis also revealed significant differences in cellular metabolism when macrophages were differentiated under microgravity. Importantly, the microgravity-related phenotypes, including poor macrophage differentiation, could be rescued by exogenous application of ERK and NFKB activators. These findings provide new insights into how microgravity affects gene transcription in developing macrophages and suggest that exogenous ERK and NFKB activators could potentially be used to prevent microgravity-caused monocyte development deficiency.

During development and activation, macrophages undergo metabolic reprogramming multiple times. ${ }^{22,31}$ In this study, we found that microgravity-inhibited glycolysis but upregulated FAO to maintain mitochondrial oxidative phosphorylation. Upregulation of glycolysis has been reported to promote macrophage survival, differentiation and polarization. ${ }^{32}$ This is consistent with our findings that microgravity is associated with decreased glycolysis, which possibly contributes to monocyte development deficiency. In addition, numerous studies have investigated inflammation-induced metabolic reprogramming in activated macrophages. ${ }^{22,33}$ It was reported that glycolysis levels decreased during the immune tolerance period ${ }^{34}$ and that proinflammatory macrophages (M1) required more glycolysis
A

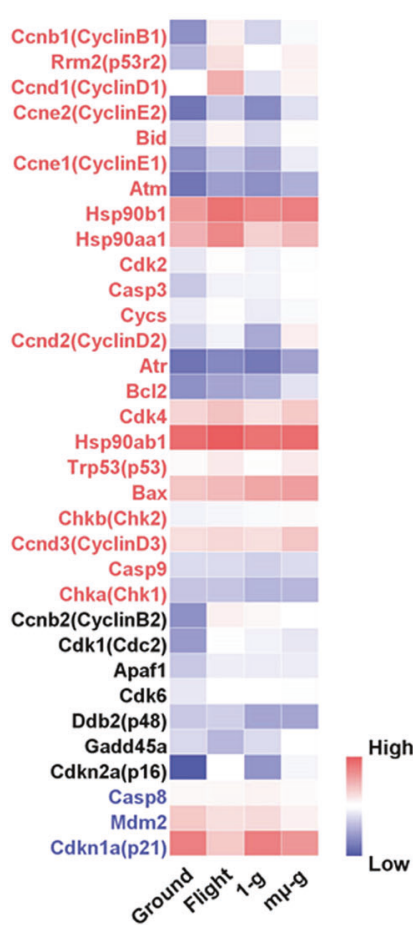

B

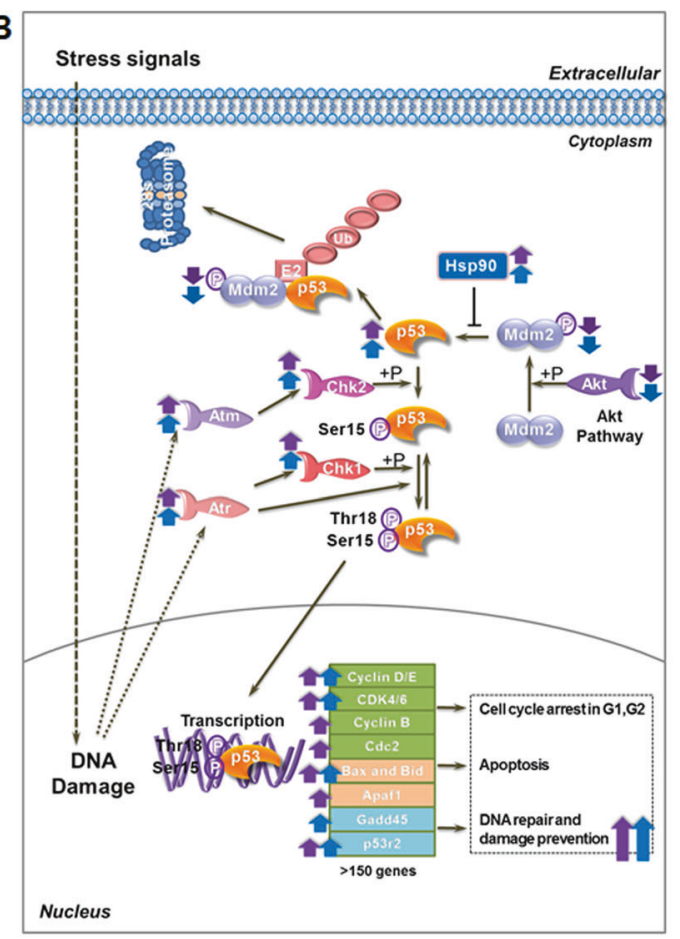

C
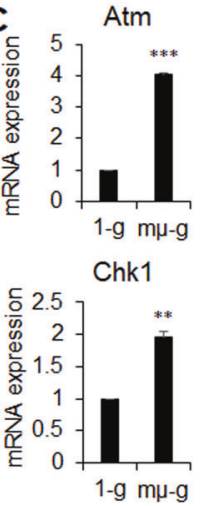

Hsp90
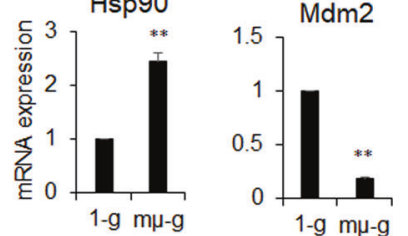

D

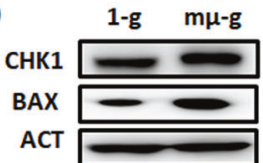


than resting macrophages. ${ }^{35,36}$ Additionally, the antiinflammatory cytokine IL-10 inhibits glycolysis and promotes oxidative phosphorylation in macrophages. ${ }^{37}$ Further, enhanced mitochondrial biogenesis and fatty acid oxidation lead to $M 2$ polarization. ${ }^{38}$ Here, we found that macrophages differentiated from HPCs under microgravity displayed fewer M2-like macrophages than macrophages differentiated under normal gravity. This result is consistent with the observed metabolic reprograming that occurs under microgravity.

The RAS/ERK/NFKB signaling network identified in our study includes two pathways, the RAS-RAF-MEK-ERK pathway and the RAS-AKT-IKK-NFKB pathway. These pathways are chains of proteins that transduce a signal from the M-CSF receptor on the surface of the cell to the DNA in the cell nucleus. ${ }^{39}$ Our NGS results showed that the expression levels of the M-CSF receptor, RAS, and the two major RAS effectors (RAF kinases regulating the MEK-ERK cascade and AKT kinases regulating the IKK and NFKB cascades) were slightly downregulated under microgravity conditions. However, the expression levels of MEK and ERK (ERK1 and ERK2), which are farther downstream in the ERK signaling pathway, were severely suppressed by real and simulated microgravity. This signaling pathway suppression eventually leads to less activation of transcription factors involved in cell differentiation and other fundamental cellular functions. ${ }^{40}$ Similarly, the MAP kinase NIK/MEKK1, IKK $1 / 2 / 3$, and IKBA in the NFKB pathway were expressed at lower levels under microgravity than under normal gravity. This suppression leads to the reduced phosphorylation, ubiquitination, and proteasomal degradation of IKBA and fewer free NFKB/REL complexes. The bound NFKB transcription factor cannot promote the expression of c-JUN, c-FOS, and some cytokines and proteins involved in the development and function of macrophages. ${ }^{41}$ In short, the ERK and NFKB pathways have been shown to play a key role in the transduction of extracellular signals, and their downregulation may underlie the macrophage phenotypes observed under microgravity.

In a complete assessment of regulatory networks, we found that pathways related to repair and degradation were significantly enriched in macrophages differentiated under microgravity. We observed that microgravity disrupted macrophage function, produced a large amount of damaged DNA and unneeded RNAs and proteins, and activated mechanisms of damage tolerance. Notably, p53 is a principal mediator of DNA repair, cell cycle arrest, and apoptosis in response to a broad array of cellular damage and is activated as a transcription factor by stress. ${ }^{42}$ Rapid induction of high p53 protein levels by various types of stress prevents the inappropriate propagation of cells that carry damaged, potentially mutagenic DNA. ${ }^{43}$ p53 protein levels are the single most important determinant of its function. ${ }^{44}$ In normal, unstressed cells, p53 is very unstable, with a half-life ranging from 5 to $30 \mathrm{~min}$. Low levels of p53 are maintained by continuous degradation, largely mediated by Mdm2, a key regulator of p53 activity and stability, and the interaction between Mdm2 and p53 leads to p53 ubiquitination and rapid degradation. In the present study, we demonstrated that microgravity upregulates the expression of $p 53$ via decreasing the expression of Mdm2. Increased p53 expression enhances cell cycle arrest, apoptosis, DNA repair, and DNA damage prevention.

Collectively, microgravity affects the RAS/ERK/NFKB and p53 pathways and cellular metabolism, leading to changes in the cellular status and function. Signaling crosstalk among these pathways is complex. p53 is reported to inhibit inflammation, which itself is an antagonist of NFKB. ${ }^{45}$ By suppressing NFKB, p53 generally suppresses innate immunity, ${ }^{45}$ and the degradation of p53 results in enhanced NFKB expression after LPS stimulation. Therefore, in our study, the microgravity-induced increase in p53 may have caused a reduction in NFKB activation during macrophage differentiation. This phenomenon should be further investigated in future studies. Additionally, research has shown that RAS/MAPK, AKT, and NFKB signals promote glycolysis, ${ }^{46-48}$ while p53 inhibits glycolysis and promotes oxidative phosphorylation. ${ }^{49}$ These glycometabolic alterations can further promote the survival, differentiation, and activation of macrophages. ${ }^{32}$ Conversely, lactic acid produced by glycolysis in macrophages can lead to increased release of inflammatory cytokines, such as IL-1 $\beta$ and HMGB1, through the NFKB signaling pathway, which affects cell division, differentiation, and self-renewal. ${ }^{50}$ Further, some tumor cells display increased lipid metabolism downstream of p53 pathway upregulation. ${ }^{51}$ Therefore, microgravity inhibition of the RAS/ERK/NFKB pathway may directly lead to the downregulation of glycolysis, as demonstrated by our research. On the other hand, induction of the p53 pathway by microgravity may cause increased lipid metabolism, which needs further study. A summary of the relationships of all signaling pathways, metabolic changes, and macrophage state changes influenced by microgravity is schematized in Supplementary Fig. 9.

Because experiments in orbit are rare and costly, studies about the biological effects of spaceflight are limited. In the current study, we not only observed macrophage differentiation under two conditions, spaceflight and ground control, but also analyzed the transcriptional profiles of macrophages under these two conditions. The state of gravity (microgravity, normogravity, and hypergravity) is complex and variable in spaceflight experiments, including spacecraft launch (at $\sim 0-8 \mathrm{~g}$ ), return, reentry and landing; however, the duration of the launch is very short $(\sim 10$ min), and numerous effective methods have been adopted for reducing the microgravity change during return, reentry and landing. ${ }^{52}$ Moreover, the effects of short-term force vibrations on macrophage development have been explored, and no significant effect was observed (data not shown). Although several forms of ground-based setups simulating "weightlessness" have been developed and applied to explore the effects of microgravity on macrophages, simulated microgravity still differs from the real state of microgravity during spaceflight. Simulated microgravity is not simply a low-gravity environment, as it also includes shear, friction, and other complex forces..$^{53-55}$ Therefore, the combined analysis of samples under both spaceflight and simulated microgravity conditions, as performed in this study, is particularly important. Our findings in simulated and space microgravity showed largely identical effects; however, these different microgravity conditions did induce some subtle differences in transcriptional profiles. Thus, the transcriptional changes caused by real microgravity (that is, during spaceflight) were determined first, and similar changes were then identified and verified in the simulated microgravity samples.

Further, isolated cells are an ideal study object to investigate direct microgravity-induced changes without any indirect or secondary influence, such as the extracellular microenvironment and structure of interaction between cells. However, the in vivo lifespan of tissue-resident macrophages ranges from several weeks to years, ${ }^{10,56}$ significantly longer than their lifespan in culture. With future technological developments, we hope to be able to perform in vivo studies.

The adaptation and changes of the monocyte/macrophage system in a microgravity environment appear to be very complex. Many questions about microgravity-regulated genes remain unanswered and warrant further investigation. Nevertheless, in this study, we have provided a more complete and comprehensive analysis of microgravity-driven changes in gene regulation networks during macrophage differentiation. We have identified several new signaling pathways with potentially important functions for macrophage differentiation under microgravity. These findings enable a better understanding of how microgravity impacts the monocyte/macrophage system and thus how spaceflight affects innate immune defense. Such insights into the biological effects of microgravity will ultimately lead to improved health outcomes for our pilots and astronauts. 


\section{MATERIALS AND METHODS}

Flight information: programs and spaceflight

This study was conducted during two flight programs, the Tianzhou1 cargo ship program $^{57}$ and the SJ-10 space program of microgravity research. ${ }^{57-61}$ All spaceflights took place in circular orbit at an altitude of $\sim 252 \mathrm{~km}$ and a flight path angle of 43 degrees. The daily average dose of radiation did not exceed $1 \times 10^{-2}$ Gy (100 mrad). With the coating control, the amount of radiation in the cabin ( $0.18 \mathrm{mGy})$ was approximately the same as that on an ordinary aircraft. $^{62}$ The main objective of the Tianzhou- 1 mission was to conduct an on-orbit refueling of the Tiangong-2 space station. At 19:41:35 p.m. on April 20, 2017, Tianzhou-1 was launched by the rocket Long March 7 at the China Wenchang Spacecraft Launch Site. Our experiment was designed to obtain information on the growth and development of cells using image acquisition. HPCs were cultured in two specialized cell observation units (Fig. 1b) and flown onboard the Tianzhou-1 cargo ship in outer space for $\sim 12$ days. We used space teleoperation technology to operate a real-time photomicrography device (10x and $20 \times$ microscopes) and capture images of macrophage differentiation from HPCs every $24 \mathrm{~h}$ after the satellite arrived at its anticipated orbit. The images were transmitted back to the ground when the satellite was circulating through Chinese airspace. At the end of the mission, HPCs in the cell culture unit were destroyed in Earth's atmosphere along with Tianzhou-1. The SJ-10 program was China's first recoverable satellite used for microgravity experiments. It was organized by the Chinese National Space Administration (CNSA) in the mid-2000s and launched at 1:38 a.m. on April 6, 2016. ${ }^{62}$ Our experiment was designed to investigate the effects of microgravity on the development of macrophages derived from mouse bone marrow HPCs. HPCs were cultured in a specialized cell culture unit (Fig. 1c) and flown on board the SJ-10 satellite in outer space for approximately 12 days. On April 18, 2016, the SJ-10 satellite returned safely to Earth, and the cultured cells were collected for our study. During landing and recovery of the SJ-10 satellite, the re-entry parachute module and the main parachute each popped up in turn, allowing re-entry to occur slowly without the influence of gravity changes. The exact experimental details, including the time, handling and sequences of the Tianzhou-1 and SJ-10 spaceflight experiments, are described in Supplementary Tables 4 and 5. All animal procedures for inflight studies were approved by the Ethics Committee of Institute of Zoology, Chinese Academy of Sciences.

\section{Cell culture and hardware specifications}

During a 12-day space mission, the sorted Lin ${ }^{-}$HPCs were cultured in special cell culture units, which can automatically replace $5 \mathrm{ml}$ of medium every day $\left(1 \mathrm{ml} / \mathrm{h}, 5 \mathrm{~h}\right.$ per day) at $37^{\circ} \mathrm{C}$ without $\mathrm{CO}_{2}$, and flew aboard the SJ-10 satellite and Tianzhou- 1 cargo ship in outer space for $\sim 12$ days. For ground controls, HPCs were cultured in the same cell culture units $\left(0 \% \mathrm{CO}_{2}\right)$ and maintained in a $37{ }^{\circ} \mathrm{C}$ incubator in parallel with the space mission.

For simulated microgravity experiments, the sorted mouse HPCs were cultured as previously described. ${ }^{21,61}$ Specifically, the HPCs were inoculated into $10 \mathrm{ml}$ culture vessels in RCCS and cultured for 12 days in a humidified $37^{\circ} \mathrm{C}$ incubator with $5 \% \mathrm{CO}_{2}$. The HPCs were also cultured in 6-well culture plates for 12 days in a humidified $37^{\circ} \mathrm{C}$ incubator with $5 \% \mathrm{CO}_{2}$ for the normal gravity condition (control group, 0 rpm). For rescue experiments, LM22B10 (an ERK activator, manufactured by MedChemExpress) and doxifluridine (an NFKB activator, manufactured by Santa Cruz Biotechnology) were added to the medium at final concentrations of $200 \mathrm{nM}$ and $3 \mu \mathrm{M}$, respectively.

All cell culture devices for spaceflight were designed and prepared by Shanghai Institute of Technical Physics, Chinese Academy of Sciences as reported previously. ${ }^{60,61,63}$ The details are as follows. The device contained four parts: cell culture units, liquid storage units, an automatic liquid change system, and an electronic control system. ${ }^{60}$ The cell observation units used on the
Tianzhou-1 cargo ship, in which $60-80$ Cytodex 3 microcarriers ${ }^{64,65}$ laden with attached HPCs (over $80 \%$ of the microcarriers were covered with cells, 5-20 cells per ball) were exposed to $1.8 \mathrm{ml}$ of medium, were separated by two pieces of polycarbonate filter membrane at each end to prevent pollution from the liquid storage units (Fig. 1b) ${ }^{66}$ The camera photographed cells from the glass observation window in the unit and collected the image information. Two units were connected in series: one imaged cells at $10 \times$ magnification, and the other imaged at $20 \times$ magnification. The medium flowed from the nutrition intake port to the waste outlet and provided nutrients for the cells in the cell culture chamber. The cell culture units used in the SJ-10 satellite, in which $2 \times 10^{6}$ cells were placed in $2.5 \mathrm{ml}$ of medium, were also designed to prevent pollution from the liquid storage units (Fig. 1c). ${ }^{63}$ The cells cultured in this unit eventually returned to the ground for further analysis. In the above two spaceflight devices, the medium entered the culture unit from the nutrient solution storage bag (polyvinyl chloride, PVC profiles) connected at one end, infiltrated the filter membrane, entered the cell culture chamber, and then passed from the other end of the culture unit to the liquid waste bag (PVC profiles). An automatic liquid change system (thermoplastic elastomer, TPE for all pipelines) and an electronic control system were used to control liquid circulation.

The RCCS was designed and prepared by Dr. Shujin Sun at the Institute of Mechanics, Chinese Academy of Sciences. The RCCS culture vessels are breathable but impermeable to liquid (Supplementary Fig. 1), which rotates in a clockwise direction at a speed of $24 \mathrm{rpm}$. The cell suspension was injected into the vessels, after which the air bubbles were carefully removed, and the medium was changed every 2-3 days. This rotary culture system, also known as a clinostat, can nullify a gravity stimulus and thus nearly perfectly mimic the effects of actual weightlessness when appropriate working conditions are established. ${ }^{54,67}$ Briefly, these conditions include (a) small particle size, (b) minimal density difference between the particle and medium, (c) sufficient rotating speed to maintain the particle within Brownian "noise", and (d) sufficiently slow speed and small container to avoid the introduction of significant centrifugal force. For cell biology experiments, some tradeoffs must be considered when the above conditions are applied, such as the rotation speed, vessel size and sample volume. In this rotary system, the radius of the culture vessel is $2 \mathrm{~cm}$, and the rotation speed is $24 \mathrm{rpm}$, generating a maximum centrifugal force of approximately $10^{-2} \mathrm{~g}$. In this situation, the density difference between the cells and medium is very small $\left(4-7 \times 10^{-2} \mathrm{~g} / \mathrm{ml}\right)$, and ${ }^{54}$ the sedimentation velocity is thus also very small, helping to keep cells within Brownian "noise" according to the formula for sedimentation velocity and maximum fluid shear of a suspension system. ${ }^{54}$ Furthermore, the fluid shear stress is also very small, approximately $10^{-4}-10^{-5} \mathrm{dyn} /$ $\mathrm{cm}^{2}$, which is below the threshold of $10^{-2} \mathrm{dyn} / \mathrm{cm}^{2}$ that is thought to have effects on cells. ${ }^{68}$ In addition, the bubble-free condition allows the avoidance of turbulence and unexpected shear force, and the low sedimentation velocity and moderate rotation speed ensures that the Coriolis force in this rotary system is very weak and negligible.

\section{Preparation and differentiation of mouse HPCs}

Male C57BL/6 mice were purchased from Beijing Vital River Experimental Animal Center (Beijing, China). The mice were maintained in pathogen-free conditions and euthanized in accordance with the "Guide for the Care and Use of Laboratory Animals" of China. Bone marrow cells were obtained from the mouse femurs, humeri, and tibias (6-8 weeks old; $n=20)$ as described previously ${ }^{69}$ and further purified using the Lin $^{-}$sorting kit (BD, \#559971). The Lin cells were then suspended in RPMI-1640 medium supplemented with $10 \%$ fetal bovine serum (Gibco), 100 $\mathrm{U} / \mathrm{ml}$ penicillin (Sigma), $0.1 \mathrm{mg} / \mathrm{ml}$ streptomycin (Sigma), $10 \mathrm{ng} / \mathrm{ml}$ IL-3 (PeproTech), 20 ng/ml IL-6 (PeproTech), and $100 \mathrm{ng} / \mathrm{ml} \mathrm{M-CSF}$ 
(PeproTech) in preparation for culture under normal gravity or microgravity.

\section{Macrophage polarization}

The macrophages developed under normal and simulated microgravity were collected and then stimulated with cytokines under normal gravity conditions to polarize them into $M 1$ and $M 2$ macrophages. ${ }^{26}$ For $\mathrm{M} 1$ macrophage polarization, the macrophages were treated with IFN- $\gamma(50 \mathrm{ng} / \mathrm{ml})$ for $26 \mathrm{~h}$, followed by stimulation with lipopolysaccharide (LPS, $100 \mathrm{ng} / \mathrm{ml}$ ) in the presence of GolgiPlug (BD Pharmingen) for the last $6 \mathrm{~h}$. For M2 polarization, macrophages were treated with IL-4 $(1000 \mathrm{U} / \mathrm{ml})$ for $48 \mathrm{~h}^{26}$

\section{Flow cytometry}

Anti-mCD11b-FITC, anti-mF4/80-PE-Cy5, anti-mTNF-a-PE, antimCD206-FITC, anti-mCD11b-PE-Cy5, anti-mF4/80-PE, anti$m C D 115-P E$, and anti-I-Ab-PE were purchased from BD Biosciences Pharmingen. Cells were directly stained with antibodies in PBS containing $0.1 \%$ BSA and $0.1 \%$ sodium azide or fixed, permeabilized and stained for the intracellular production of cytokines. ${ }^{31}$ The stained samples were subsequently assayed using a FC500 fluorescence-activated cell sorting (FACS) instrument (Beckman Coulter, USA).

Quantitative real-time RT-PCR analysis

Total cellular RNA was extracted from the differentiated and subsequently polarized macrophages using an EZNA MicroElute Total RNA Kit (Omega Bio-Tek). ${ }^{70}$ The reverse transcription reaction was performed with oligo (dT) primers and AMV reverse transcriptase XL (Takara Bio, Shiga, Japan), and real-time PCR was performed using TB Green Premix Ex Taq (Takara Bio, Shiga, Japan). All primers used in the present study are listed in Supplementary Table 6 . To assess the relative induction of cytokines and marker molecule mRNA expression in response to various stimuli, the mRNA expression levels of each gene were normalized to that of the housekeeping gene Hprt (hypoxanthine phosphoribosyl transferase) or $\beta$-actin.

\section{Western blotting}

Western blot analysis was performed on total proteins extracted from samples as previously described. ${ }^{71}$ Proteins were harvested by lysing collected cells with $200 \mathrm{ml}$ of RIPA buffer containing protease inhibitor and phosphatase inhibitor cocktail tablets (Roche, Brussels, Belgium). Western blotting was performed using standard procedures with the following primary antibodies: c-FOS (9F6) rabbit mAb (catalog number 2250), c-JUN (60A8) rabbit mAb (catalog number 9165), phosphor-c-FOS (Ser32) rabbit mAb (D82C112), phosphor-c-JUN (Ser73) rabbit mAb (D47G9), NFKB p65 (L8F6) mouse mAb (catalog number 6596), phosphor- NFKB p65 (Ser468) (catalog number 3039), and $\beta$-Actin (13E5) rabbit mAb (catalog number 4970) used as a loading control from Cell Signaling Technology (Leiden, The Netherlands); ACSL3 (sc166374) from Santa Cruz; and IKBA (ab32518), Bax (ab32503), Hexokinase 1 (ab150423), Chk1 (ab32531), PFKP (ab204131), ERK1/ 2 (ab184699), and p-ERK1/2 (ab201015) from Abcam (China). For visualization, we used Immobilon Western Chemiluminescent HRP Substrate (WBKLS0500, Millipore).

\section{Immunofluorescence staining}

The cells that differentiated during the spaceflight were collected, fixed and stained as previously reported. ${ }^{62}$ Photomicrographs were taken using the LSM510META laser scanning microscope (Zeiss, Germany). Anti-mF4/80-FITC (1:500 dilution in blocking buffer) and Hoechst $33342(2 \mu \mathrm{g} / \mathrm{ml})$ were applied.

Whole transcriptomic analysis by RNA-Seq

Whole transcriptomic analysis was carried out on macrophages following in vitro differentiation in real and simulated microgravity and on the corresponding control conditions. Then, the data were analyzed using the corresponding $\mathrm{R}$ package. ${ }^{72-77}$ Detailed information is provided in the online supplementary materials.

\section{Metabolism assay}

Metabolic differences in macrophages following in vitro differentiation in simulated microgravity and the corresponding control conditions were detected by Seahorse Bioscience XFe24 Extracellular Flux Analyzers (Seahorse Bioscience, USA). ${ }^{78}$ Specifically, the treated macrophages were seeded into XF24 cell culture microplates (Seahorse Bioscience) with cell-tak and preequilibrated for $1 \mathrm{~h}$ in unbuffered XF assay medium supplemented with $2.05 \mathrm{mM}$ glutamine (glycolysis assessments) or $11.11 \mathrm{mM}$ glucose and $2.05 \mathrm{mM}$ glutamine (fatty acid oxidation). For glycolysis assessments, compounds were injected during the assay according to the manufacturer's protocol at the following final concentrations: $10 \mathrm{mM}$ glucose, $1 \mu \mathrm{M}$ oligomycin, and $50 \mathrm{mM}$ 2-DG. For cellular FAO quantification, the XF Mito fuel flex test kit was used according to the manufacturer's protocol. The extracellular acidification rate (ECR) and oxygen consumption rate (OCR) were examined with XFe24 Analyzer (Seahorse Bioscience), and XFe Wave software (Seahorse Bioscience) was used to analyze the results.

\section{Statistical analysis}

All experiments except RNA-Seq were conducted more than three times, and the data are presented as the mean \pm SD of three independent experiments. Differences between groups were calculated using Student's unpaired $t$-test. $P$-values $<0.05$ were regarded as significant, and $P$-values $<0.01$ were regarded as highly significant.

\section{DATA AVAILABILITY}

The raw RNA-Seq data from this study were deposited into the BIGD Genome Sequence Archive (GSA) under accession number CRA000920.

\section{ACKNOWLEDGEMENTS}

The authors appreciate Drs. Yuzhu Hou and Peng Wang for their critical review of our manuscript. This work was supported by grants from the National Key Research and Development Program of China (2017YFA0105002, Y.Z., 2017YFA0104402, L.L.), Joint Funds of the National Natural Science Foundation of China (U1738111, Y.Z.), the China Manned Space Flight Technology Project (TZ-1), and the National Natural Science Foundation Youth Fund (31800741, L.S.).

\section{AUTHOR CONTRIBUTIONS}

Y.Z., H.T., and L.S. conceived the study and designed the experiments. L.S. analyzed and interpreted all the data and drafted the manuscript with input from all coauthors. H.T. prepared the mouse HPCs and performed the cell experiments. P.W. conducted the molecular experiments. L.L., Z.Z., and J.Z. processed the raw data from NGS. Y.Z. supervised the project and provided critical feedback at all stages.

\section{ADDITIONAL INFORMATION}

The online version of this article (https://doi.org/10.1038/s41423-019-0346-6) contains supplementary material.

Competing interests: The authors declare no competing interests.

\section{REFERENCES}

1. Grigor'ev, A. I. Physiological problems of manned mission to Mars. Ross. Fiziol. Zh . Im. I M Sechenova. 93, 473-484 (2007).

2. Wichman, H. A. Behavioral and health implications of civilian spaceflight. Aviat. Space Environ. Med. 76(6 Suppl), B164-B171 (2005).

3. Davis, T. A. et al. Effect of spaceflight on human stem cell hematopoiesis: suppression of erythropoiesis and myelopoiesis. J. Leukoc. Biol. 60, 69-76 (1996). 
Spaceflight and simulated microgravity suppresses macrophage development...

4. Blaber, E., Marcal, H. \& Burns, B. P. Bioastronautics: the influence of microgravity on astronaut health. Astrobiology 10, 463-473 (2010)

5. Hughes-Fulford, M., Chang, T. T., Martinez, E. M. \& Li, C. F. Spaceflight alters expression of microRNA during T-cell activation. FASEB J. 29, 4893-4900 (2015).

6. Kraemer, W. J. et al. Responses of plasma proenkephalin peptide $\mathrm{F}$ in rats following 14 days of spaceflight. Aviat. Space Environ. Med. 75, 114-117 (2004).

7. Hwang, S. A., Crucian, B., Sams, C. \& Actor, J. K. Post-spaceflight (STS-135) mouse splenocytes demonstrate altered activation properties and surface molecule expression. PLoS ONE 10, e0124380 (2015).

8. Paulsen, K. et al. Regulation of ICAM-1 in cells of the monocyte/macrophage system in microgravity. Biomed. Res Int. 2015, 538786 (2015).

9. Taylor, G. R., Konstantinova, I., Sonnenfeld, G. \& Jennings, R. Changes in the immune system during and after spaceflight. Adv. Space Biol. Med. 6, 1-32 (1997).

10. Tauber, S. et al. Cytoskeletal stability and metabolic alterations in primary human macrophages in long-term microgravity. PLoS ONE 12, e0175599 (2017).

11. Stowe, R. P., Sams, C. F. \& Pierson, D. L. Adrenocortical and immune responses following short- and long-duration spaceflight. Aviat. Space Environ. Med. 82, 627-634 (2011)

12. Crucian, B. E., Stowe, R. P., Pierson, D. L. \& Sams, C. F. Immune system dysregulation following short- vs long-duration spaceflight. Aviat. Space Environ. Med. 79, 835-843 (2008)

13. Girardi, $C$. et al. Integration analysis of microRNA and mRNA expression profiles in human peripheral blood lymphocytes cultured in modeled microgravity. Biomed. Res Int. 2014, 296747 (2014).

14. Tauber, S. et al. Signal transduction in primary human T lymphocytes in altered gravity during parabolic flight and clinostat experiments. Cell Physiol. Biochem. 35, 1034-1051 (2015)

15. Baqai, F. P. et al. Effects of spaceflight on innate immune function and antioxidant gene expression. J. Appl Physiol. 106, 1935-1942 (2009).

16. Crucian, B., Stowe, R., Quiriarte, H., Pierson, D. \& Sams, C. Monocyte phenotype and cytokine production profiles are dysregulated by short-duration spaceflight. Aviat. Space Environ. Med. 82, 857-862 (2011).

17. Hsieh, C. L., Chao, P. D. \& Fang, S. H. Morin sulphates/glucuronides enhance macrophage function in microgravity culture system. Eur. J. Clin. Invest. 35, 591-596 (2005)

18. Wang, C. et al. Microgravity inhibition of lipopolysaccharide-induced tumor necrosis factor-alpha expression in macrophage cells. Inflamm. Res. 63, 91-98 (2014).

19. Armstrong, J. W., Gerren, R. A. \& Chapes, S. K. The effect of space and parabolic flight on macrophage hematopoiesis and function. Exp. Cell Res. 216, 160-168 (1995).

20. Ullrich, O., Huber, K. \& Lang, K. Signal transduction in cells of the immune system in microgravity. Cell Commun. Signal. 6, 9 (2008).

21. Wang, C. et al. Microgravity activates p38 MAPK-C/EBPbeta pathway to regulate the expression of arginase and inflammatory cytokines in macrophages. Inflamm. Res. 64, 303-311 (2015).

22. Zhu, L., Zhao, Q., Yang, T., Ding, W. \& Zhao, Y. Cellular metabolism and macrophage functional polarization. Int. Rev. Immunol. 34, 82-100 (2015).

23. Murray, P. J. et al. Macrophage activation and polarization: nomenclature and experimental guidelines. Immunity 41, 14-20 (2014).

24. Martinez, F. O., Sica, A., Mantovani, A. \& Locati, M. Macrophage activation and polarization. Front. Biosci. 13, 453-461 (2008).

25. Hou, Y. et al. IL-23-induced macrophage polarization and its pathological roles in mice with imiquimod-induced psoriasis. Protein Cell 9, 1027-1038 (2018).

26. Zhu, L. et al. TSC1 controls macrophage polarization to prevent inflammatory disease. Nat. Commun. 5, 4696 (2014).

27. Krausgruber, T. et al. IRF5 promotes inflammatory macrophage polarization and TH1-TH17 responses. Nat. Immunol. 12, 231-238 (2011).

28. Gundra, U. M. et al. Alternatively activated macrophages derived from monocytes and tissue macrophages are phenotypically and functionally distinct. Blood 123, e110-e122 (2014).

29. Tanabe, M. \& Kanehisa, M. Using the KEGG database resource. Curr. Protoc. Bioinformatics 38, 1.12.1-1.43 (2012).

30. Sabapathy, K. \& Lane, D. P. Therapeutic targeting of p53: all mutants are equal, but some mutants are more equal than others. Nat. Rev. Clin. Oncol. 15, 13-30 (2018).

31. Zhao, Y. et al. mTOR masters monocyte development in bone marrow by decreasing the inhibition of STAT5 on IRF8. Blood 131, 1587-1599 (2018).

32. Kornberg, M. D. et al. Dimethyl fumarate targets GAPDH and aerobic glycolysis to modulate immunity. Science 360, 449-453 (2018).

33. Zhao, Q. et al. 2-Deoxy-d-Glucose treatment decreases anti-inflammatory $M 2$ macrophage polarization in mice with tumor and allergic airway inflammation. Front Immunol. 8, 637 (2017).

34. Wang, C., Bo, L. \& Deng, X. Research progress on metabolism of monocytes and macrophages in sepsis. Zhonghua Wei Zhong Bing. Ji Jiu Yi Xue. 29, 381-384 (2017)
35. Kannan, Y. et al. TPL-2 regulates macrophage lipid metabolism and M2 differentiation to control TH2-mediated immunopathology. PLoS Pathog. 12, e1005783 (2016).

36. Mills, E. L. et al. Succinate dehydrogenase supports metabolic repurposing of mitochondria to drive inflammatory macrophages. Cell 167, 457-70 e13 (2016).

37. Ip, W. K. E., Hoshi, N., Shouval, D. S., Snapper, S. \& Medzhitov, R. Anti-inflammatory effect of IL-10 mediated by metabolic reprogramming of macrophages. Science 356, 513-519 (2017).

38. Miao, L. et al. Hydrogen sulfide mitigates myocardial infarction via promotion of mitochondrial biogenesis-dependent M2 polarization of macrophages. Antioxid. Redox Signal. 25, 268-281 (2016).

39. Yoshida, S. et al. Differential signaling during macropinocytosis in response to $M$ CSF and PMA in macrophages. Front. Physiol. 6, 8 (2015).

40. Avruch, J. et al. Ras activation of the Raf kinase: tyrosine kinase recruitment of the MAP kinase cascade. Recent Prog. Horm. Res. 56, 127-155 (2001).

41. Smith, E. M., Gregg, M., Hashemi, F., Schott, L. \& Hughes, T. K. Corticotropin releasing factor (CRF) activation of NF-kappaB-directed transcription in leukocytes. Cell Mol. Neurobiol. 26, 1021-1036 (2006).

42. Yoshida, K. \& Miki, Y. The cell death machinery governed by the p53 tumor suppressor in response to DNA damage. Cancer Sci. 101, 831-835 (2010).

43. Dregoesc, D., Rybak, A. P. \& Rainbow, A. J. Increased expression of p53 enhances transcription-coupled repair and global genomic repair of a UVC-damaged reporter gene in human cells. DNA Repair 6, 588-601 (2007).

44. Herce, H. D., Deng, W., Helma, J., Leonhardt, H. \& Cardoso, M. C. Visualization and targeted disruption of protein interactions in living cells. Nat. Commun. 4, 2660 (2013).

45. Machado, D. et al. Role of p53/NF-kappaB functional balance in respiratory syncytial virus-induced inflammation response. J. Gen. Virol. 99, 489-500 (2018).

46. Peeters, K. et al. Fructose-1,6-bisphosphate couples glycolytic flux to activation of Ras. Nat. Commun. 8, 922 (2017).

47. Griffiths, H. R., Gao, D. \& Pararasa, C. Redox regulation in metabolic programming and inflammation. Redox Biol. 12, 50-57 (2017).

48. Baselga, J. Targeting the phosphoinositide-3 (PI3) kinase pathway in breast cancer. Oncologist 16(Suppl 1), 12-19 (2011).

49. Gomes, A. S., Ramos, H., Soares, J. \& Saraiva, L. p53 and glucose metabolism: an orchestra to be directed in cancer therapy. Pharm. Res. 131, 75-86 (2018).

50. Xie, M. et al. PKM2-dependent glycolysis promotes NLRP3 and AIM2 inflammasome activation. Nat. Commun. 7, 13280 (2016).

51. Li B. Immune metabolism in health and tumor (New York, NY, Springer Berlin Heidelberg, 2017).

52. Zhao H., Qiu J., Wang Y. System design and flight results of China SJ-10 recoverable microgravity experimental satellite. In Life Science in Space: Experiments on Board the SJ-10 Recoverable Satellite (eds. Duan E., Long M.) 9-42 (Springer, Singapore, 2019).

53. Consolo, F. et al. Computational modeling for the optimization of a cardiogenic 3D bioprocess of encapsulated embryonic stem cells. Biomech. Model. Mechanobiol. 11, 261-277 (2012).

54. Hammond, T. G. \& Hammond, J. M. Optimized suspension culture: the rotatingwall vessel. Am. J. Physiol. Ren. Physiol. 281, F12-F25 (2001).

55. Marsano, A. et al. Use of hydrodynamic forces to engineer cartilaginous tissues resembling the non-uniform structure and function of meniscus. Biomaterials 27, 5927-5934 (2006).

56. Murphy, J., Summer, R., Wilson, A. A., Kotton, D. N. \& Fine, A. The prolonged life-span of alveolar macrophages. Am. J. Respir. Cell Mol. Biol. 38, 380-385 (2008).

57. Meng, D. \& Jianjun, X. Tianzhou 1 cargo spacecraft leaves orbit. Aerosp. China 3, 62 (2017).

58. Zhao, H., Qiu, J., Tang, B., Kang, Q. \& Hu, W. The SJ-10 recoverable microgravity satellite of China. J. Space Exploration. 5, 101 (2016).

59. $\mathrm{Hu}, \mathrm{W}$. et al. Space experiments onboard the microgravity satellite SJ-10. Microgravity Sci. Technol. 26, 159-169 (2014).

60. Zhang, C. et al. Space microgravity drives transdifferentiation of human bone marrow-derived mesenchymal stem cells from osteogenesis to adipogenesis. FASEB J. 32, 4444-4458 (2018).

61. Wang, P. et al. Spaceflight/microgravity inhibits the proliferation of hematopoietic stem cells by decreasing Kit-Ras/CAMP-CREB pathway networks as evidenced by RNA-Seq assays. FASEB J. 33, 5903-5913 (2019).

62. Hu, W. R. et al. Space program SJ-10 of microgravity research. Microgravity Sci. Technol. 26, 159-169 (2014).

63. Zhang M., et al. Facilities and techniques of space life science. In Life Science in Space: Experiments on Board the SJ-10 Recoverable Satellite (ed. Duan E., Long M.) 361-398 (Springer Singapore, 2019).

64. Liu, P. C. et al. Transfection of the IHH gene into rabbit BMSCs in a simulated microgravity environment promotes chondrogenic differentiation and inhibits cartilage aging. Oncotarget 7, 62873-62885 (2016). 
65. Odeleye, A. O. O., Castillo-Avila, S., Boon, M., Martin, H. \& Coopman, K. Development of an optical system for the non-invasive tracking of stem cell growth on microcarriers. Biotechnol. Bioeng. 114, 2032-2042 (2017).

66. Lei, X. et al. Effect of microgravity on proliferation and differentiation of embryonic stem cells in an automated culturing system during the TZ-1 space mission. Cell Prolif. 51, e12466 (2018).

67. Klaus, D. M., Todd, P. \& Schatz, A. Functional weightlessness during clinorotation of cell suspensions. Adv. Space Res. 21, 1315-1318 (1998).

68. Begley, C. M. \& Kleis, S. J. The fluid dynamic and shear environment in the NASA/ JSC rotating-wall perfused-vessel bioreactor. Biotechnol. Bioeng. 70, 32-40 (2000).

69. Liu, G. et al. An instructive role of donor macrophages in mixed chimeras in the induction of recipient CD4(+)Foxp3(+) Treg cells. Immunol. Cell Biol. 89, 827-835 (2011).

70. Yang, T. et al. TSC1 controls IL-1beta expression in macrophages via mTORC1dependent C/EBPbeta pathway. Cell Mol. Immunol. 13, 640-650 (2016).

71. Sun, B. et al. Characterization and allergic role of IL-33-induced neutrophil polarization. Cell Mol. Immunol. 15, 782-793 (2018).
72. Yu, G. C., Wang, L. G., Han, Y. Y. \& He, Q. Y. clusterProfiler: an R package for comparing biological themes among gene clusters. Omics 16, 284-287 (2012).

73. Yamada, T., Letunic, I., Okuda, S., Kanehisa, M. \& Bork, P. iPath2.0: interactive pathway explorer. Nucleic Acids Res. 39(Web Server issue), W412-W415 (2011).

74. Xie, C. et al. KOBAS 2.0: a web server for annotation and identification of enriched pathways and diseases. Nucleic Acids Res. 39(Web Server issue), W316-W322 (2011).

75. Wang, L., Feng, Z., Wang, X., Wang, X. \& Zhang, X. DEGseq: an R package for identifying differentially expressed genes from RNA-seq data. Bioinformatics 26, 136-138 (2010).

76. Walter, W., Sanchez-Cabo, F. \& Ricote, M. GOplot: an R package for visually combining expression data with functional analysis. Bioinformatics 31, 2912-2914 (2015).

77. Schep, A. N. \& Kummerfeld, S. K. iheatmapr: interactive complex heatmaps in R. J. Open Source Softw. 2, 359 (2017).

78. Moon, J. S. et al. NOX4-dependent fatty acid oxidation promotes NLRP3 inflammasome activation in macrophages. Nat. Med. 22, 1002-1012 (2016). 